\title{
Ultrafast Stimulated Emission Microscopy of Single Nanocrystals
}

\author{
Lukasz Piatkowski1 ${ }^{1+*}$, Nicolò Accanto1‡\#, Gaëtan Calbris ${ }^{1 \ddagger}$, Sotirios Christodoulou ${ }^{1,2}$ \\ Iwan Moreels 2,3 and Niek F. van Hulst ${ }^{1,4 *}$
}

${ }^{1}$ ICFO - Institut de Ciences Fotoniques, the Barcelona Institute of Science and Technology, 08860 Castelldefels (Barcelona), Spain

2 Istituto Italiano di Tecnologia, Via Morego 30, 16163 Genova, Italy

3 Department of Chemistry, Ghent University, Krijgslaan 281-S3, 9000 Gent, Belgium

${ }^{4}$ ICREA-Institució Catalana de Recerca i Estudis Avançats, 08010 Barcelona, Spain

† Present address: Institute of Physical Chemistry, Polish Academy of Sciences, Kasprzaka 44/52, 01-224

Warsaw, Poland

\# Present address: Neurophotonics Lab., Paris Descartes University, 75270 Paris Cedex 06, France.

₹ These authors contributed equally to this work.

*E-mail: lukasz.j.piatkowski@put.poznan.pl; Niek.vanHulst@ICFO.eu

\begin{abstract}
The ability to detect single molecules is a powerful method used to distinguish different species and follow time trajectories hidden in the ensemble average. However, detection of singles requires efficient emitters and is prone to photobleaching, while the slow, nanosecond spontaneous emission only reports on the lowest excited state, missing out on the rich dynamics in the excited state manifold. We demonstrate direct detection of stimulated emission from individual colloidal nanocrystals at room temperature, while simultaneously recording the depleted spontaneous emission. The coherent femtosecond stimulated emission enables us to trace the carrier population through the entire photocycle. By capturing the femtosecond evolution of the stimulated emission signal, together with the nanosecond fluorescence, we disentangle the ultrafast charge trajectories in the excited state and determine the populations that experience stimulated emission, spontaneous emission and excited state absorption processes.
\end{abstract}

Complex physical, chemical and biological processes are determined by fundamental spatial and temporal interaction trajectories. Only ultrafast techniques with single-emitter sensitivity are able to unveil their inherent transient intermediates and allow exploration of processes such as molecular vibrations and energy transfer (1-3), and nanoscale dynamics in plasmonic or 2D materials $(4,5)$. The small interaction cross-sections of individual nanoparticles make it hard to rely on the conventional ultrafast approaches, such as transient absorption and nonlinear four wave mixing. Consequently, single molecules and nanoparticles are almost exclusively detected through Stokes shifted spontaneous emission (fluorescence or photoluminescence (PL)), which is background-free, allowing photon counting sensitivity, and detection of weakly fluorescent emitters. The use of fluorescence detection, however, is hampered by a number of limitations: it is restricted to luminescent samples, sensitive to bleaching, and in the linear regime it is slow (nanoseconds), reporting only on the population of the final emitting state, while missing out on femtosecond dynamics. Despite the exploration of several alternative detection schemes, such as, photo-thermal $(6)$, linear absorption $(7,8)$ or enhanced Raman (9), ultrafast detection of single entities beyond fluorescence has remained challenging.

Here we demonstrate a highly sensitive experimental scheme based on the direct detection of stimulated emission (SE) for studying the excited state dynamics in nanoscopic samples with femtosecond temporal resolution. SE microscopy involves one laser pulse for promotion to the excited state, and a second, delayed pulse, for stimulation back to the ground state, generating a new SE photon (10). SE forms the basis of the stimulated emission depletion (STED) microscopy, however, in a typical STED experiment the stimulated photons are discarded and only PL is recorded. Yet, the instantaneous SE photons contain a plethora of information on the excited state population, its dynamics and relaxation 
pattern, which is otherwise inaccessible from the slow PL. To its advantage, SE is not critical on the quantum efficiency of the sample, has femtosecond temporal resolution, is coherent, and capable of mapping the dynamics of an arbitrary excited state.

We present direct stimulated emission detection and imaging of individual NCs, and trace the excited state dynamics of single colloidal CdSe/CdS rod-in-rod NCs (11) with femtosecond temporal resolution, at ambient conditions. The PL is detected simultaneously with the SE, generating two independent, complementary images. It is important to understand the dynamic interplay between various charge relaxation pathways, such as charge injection, extraction, transfer and delocalization, and excited state relaxation, both ultrafast and with nanoscopic sensitivity (12-14). Our femtosecond SE experiment on single-NCs, shows the excited state relaxation dynamics of individual charges, the dynamical heterogeneity of NCs and the relative contributions of the various stimulated processes, all with single-NC sensitivity.

A

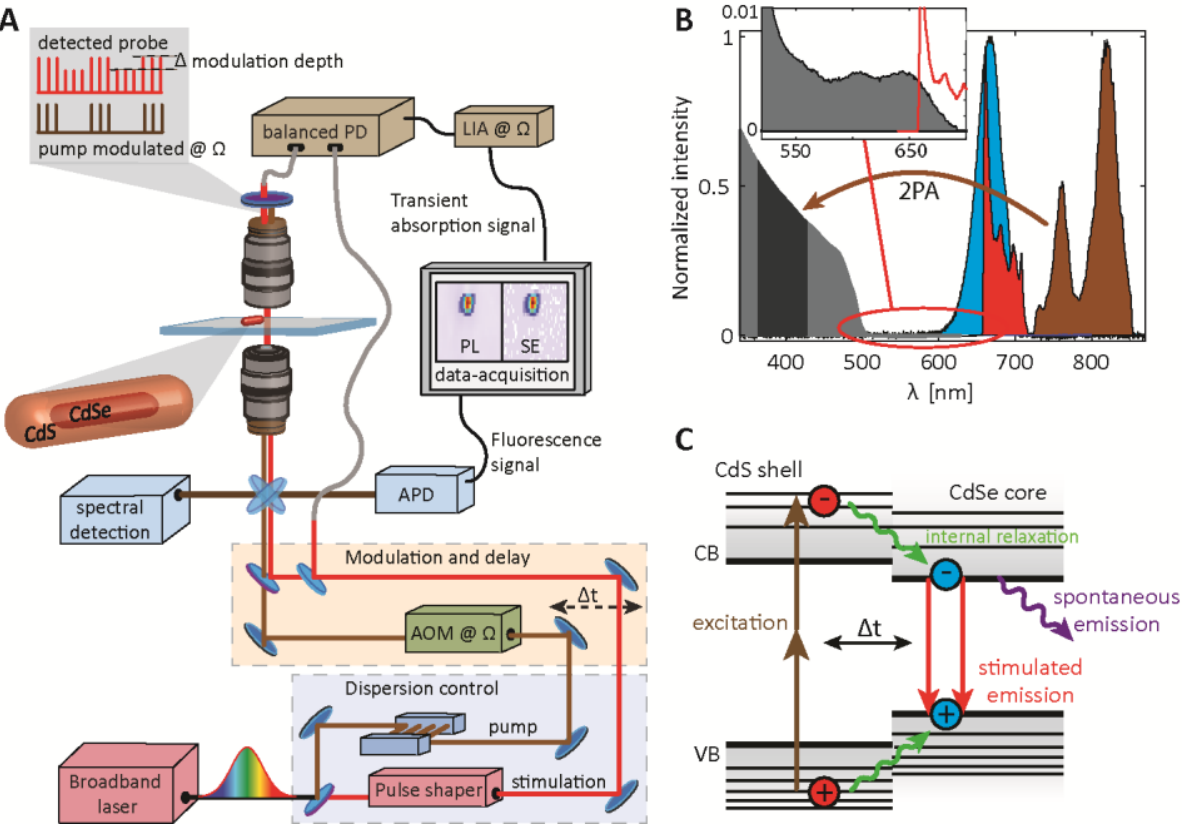

Fig. 1. Concept of the ultrafast stimulated emission nanoscopy. (A) Schematic of the experimental setup. (B) Spectral characteristics of the broadband laser pulse (pump pulse - brown, probe pulse - red) and CdSe/CdS NCs. Grey and blue shaded areas represent absorption and emission spectrum of the NCs, respectively. The black area indicates the spectral range of the two-photon absorption. (C) Energy level sketch of a core/shell CdSe/CdS NC.

In the experiment, the pump beam excites the NC through two-photon absorption to a highly excited state in the conduction band (Fig. 1, details in Materials and Methods (15)). The excited hot electrons and holes, initially localized in the shell, decay through the excited state progression and eventually localize in the lowest excited state (band edge) in the core (Fig. 1C). The probe (stimulation) beam, resonant with the core band-edge transition, leads to charge recombination, stimulates the NC back to the ground state and induces emission of a stimulated photon. Therefore, any information on the excited charges imprinted by the pump beam in the shell is 'read out' by the stimulating probe beam, when one of the excited charges reaches the core band-edge states. The pump beam is modulated, and the SE signal is retrieved by lock-in detection.

In a first step, we raster-scanned the sample while simultaneously detecting both modulated signal $\left(\mathrm{S}_{\mathrm{mod}}\right)$ and PL (Fig. 2A,B). The PL image clearly reveals the NC presence, which we verified through their emission spectra (Fig. S1). The corresponding $S_{\text {mod }}$ image shows contrast at the same sample positions where the PL signal appears. Moreover, the measured $S_{\text {mod }}$ signal was always positive, meaning we detected extra photons in our stimulation beam (Supp. Text 1). Two effects can in principle lead to an increase of the transmitted probe beam intensity when the NC is excited: stimulated emission and 
ground state depletion (GSD). In the first case, the SE process following electron-hole recombination gives a net increase in the probe beam intensity. In the second case, the absorption of the probe beam is lower because of the depletion of the ground state, due to the presence of either hole or electron in their respective energy levels. The two contributions can be readily distinguished by time-resolved experiments, as shown later. For most NCs we found a perfect correspondance between PL and $S_{\bmod }$ images and observed $S_{\text {mod }}$ wherever PL appeared (Fig. 2C). Interestingly, in some cases we detected PL, but no measurable $S_{\text {mod }}$ (Fig. 2E). We assigned this signal to core-free CdS shell nanoparticles that conucleated during synthesis. Finally, on rare ocassions we observed $S_{\text {mod }}$ contrast but no PL (Fig. 2D). The signal likely originated from highly quenched NCs, as it is improbable that we observe other particles with the exact same spectral signature. Clearly, the spectral dependence of $S_{\text {mod }}$ selected with the probe beam, and the ability to detect simultaneously PL and $S_{\text {mod }}$ gives us extra insight as to the nature of the detected NCs.

A
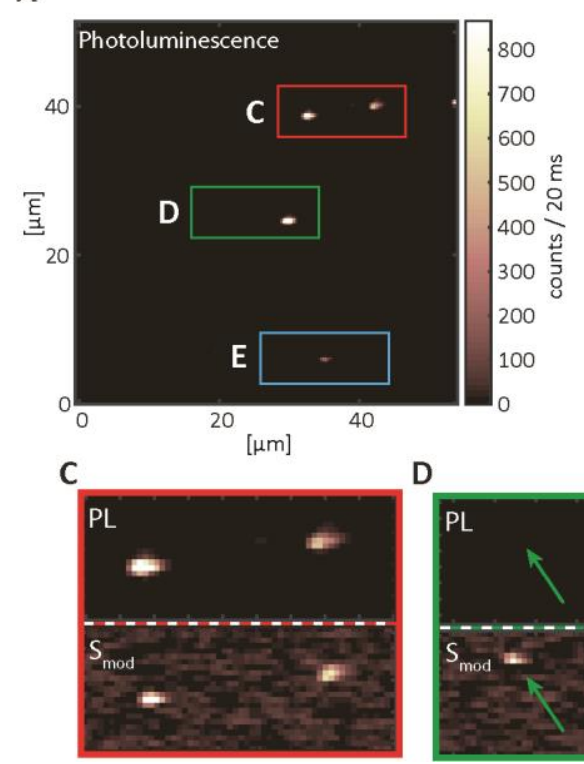

B
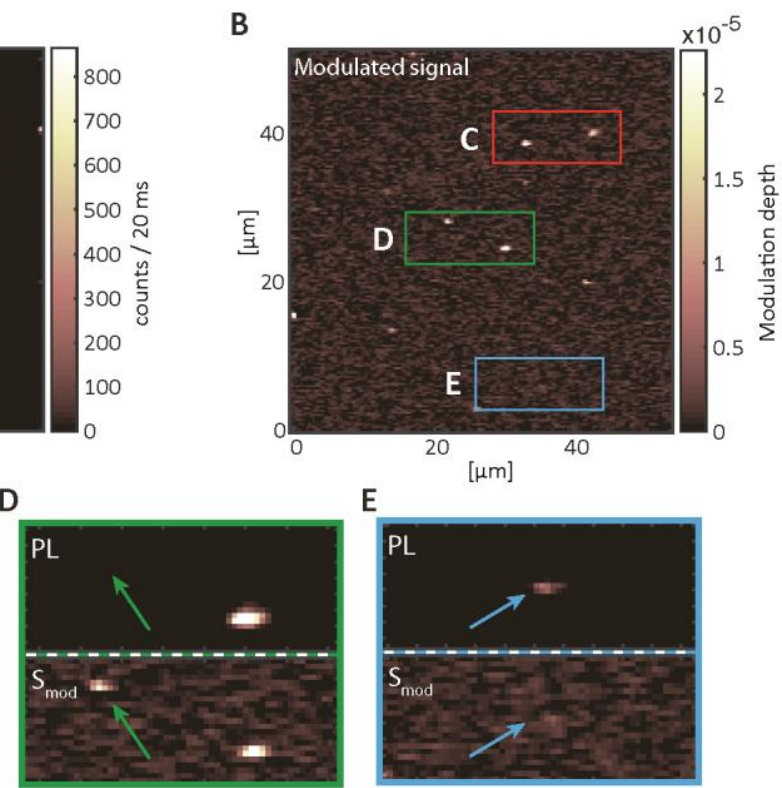

E

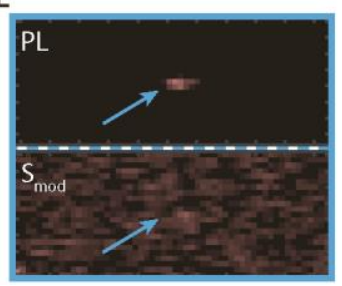

Fig. 2. Stimulated emission imaging. (A, B) Confocal images of the same sample area showing PL and lock-in signal $\left(\mathrm{S}_{\mathrm{mod}}\right)$, respectively. The stimulation beam was set to arrive 7 ps after the pump beam (Supp. Text 2). (CE) Comparison between the PL and $S_{\text {mod }}$ images for the three regions of interest marked in panels $\mathbf{A}$ and $\mathbf{B}$.

Ultrafast coherent response is the main advantage of SE detection. In Figure 3A we show a series PL and $S_{\text {mod }}$ images for different interpulse-delay (more images in Fig. S2). While PL signal is detected at all time delays $\Delta t$, the $S_{\text {mod }}$ signal appears only when the pump pulse overlaps or precedes the stimulation pulse. At negative delay times, when the stimulation pulse arrives before the pump pulse, the NC is in its ground state and there is no excited state population for the probe pulse to interact with. For the NC marked with $x$ the second order autocorrelation trace exhibits a dip with $g^{(2)}(0) \lesssim 0.5$, indicating the nonclassical emission of a single NC (Fig. S3). The time-resolved traces revealed that when $S_{\text {mod }}$ (blue) increases in time, the PL (red) decreases (Fig. 3B). This is intuitive - the excited state population, which is stimulated down back to the ground state does not contribute to the spontaneous emission, leading to PL depletion. The fact that $S_{\text {mod }}$ and PL signals are anti-correlated unambiguously indicates that $S_{\text {mod }}$ contains a significant contribution from the SE process. Furthermore, we found that the changes of both signals: $\mathrm{S}_{\text {mod }}$ ingrowth $\left(\Delta \mathrm{S}_{\bmod }\right)$ and PL depletion $(\Delta \mathrm{PL})$, occur on specific timescales. Interestingly, the $\Delta \mathrm{PL}$ depletion occurs with a single time constant, while $\Delta \mathrm{S}_{\text {mod }}$ grows in with two time constants. The slower time constant of $\sim 400-700 \mathrm{fs}$ is identical to the time with which $\Delta \mathrm{PL}$ decreases. However, a significant part of the $S_{\text {mod }}$ grows on a faster timescale, and cannot be observed within the cross-correlation time of the pump and probe pulses ( $<200 \mathrm{fs}$ ). To understand this, one needs to consider that the NCs are initially pumped to a highly excited state in the shell (Supp. Text 3), while the stimulation pulse probes the lowest 
excited state in the core. GSD occurs when charges are present in the excited state of the transition resonant with the probe energy. As soon as the faster of the two charges reaches the lowest excited state of the core (16-19), the probe beam absorption will decrease. This means that GSD reports on the relaxation rate of the fastest charge, either the electron or the hole. In contrast, the probe beam can induce charge recombination and SE only when both electron and hole localize into the core. Consequently, SE is sensitive to relaxation of the slower of the two charges. In the PL we see only the slower component, because PL is a time averaged signal, which is mostly sensitive to the population decay of the lowest excited state (Supp. Text 4).

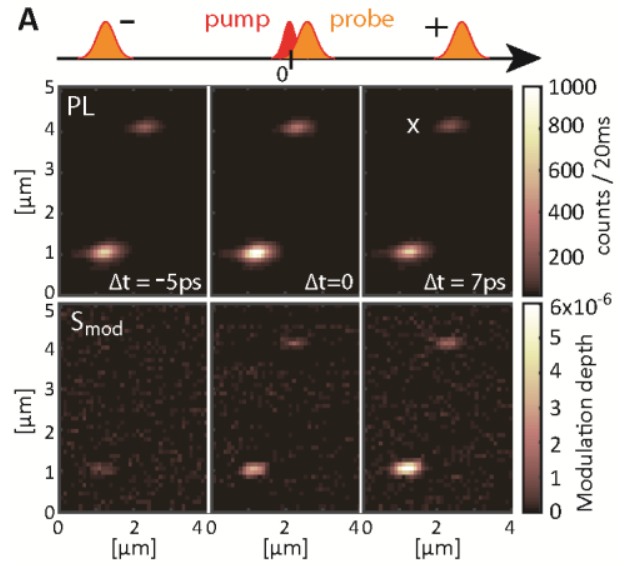

B
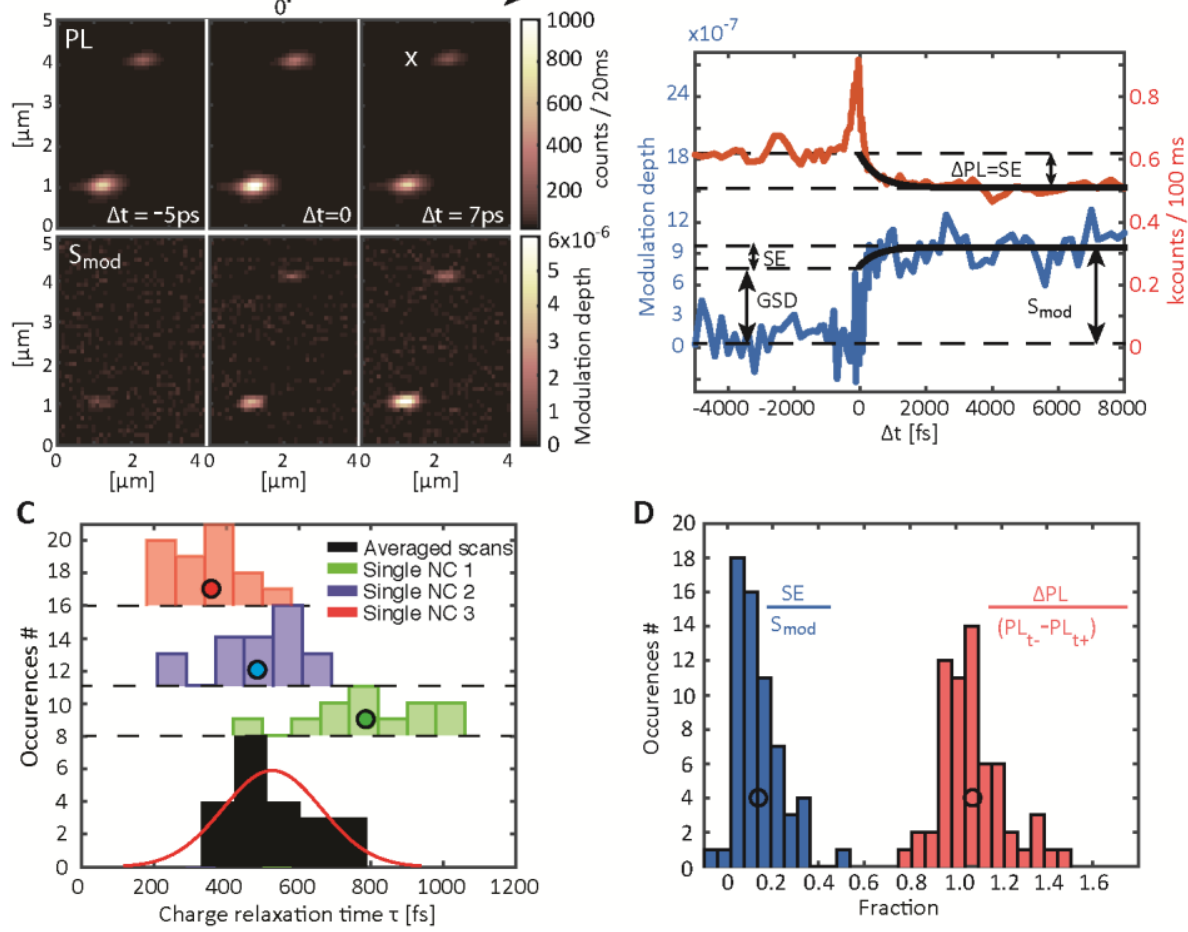

Fig. 3. Time-resolved stimulated emission microscopy. (A) A series of images acquired by detecting PL and $S_{\text {mod }}$ signal, for different excitation and stimulation interpulse delays $\Delta t$. (B) Simultaneously detected $S_{\text {mod }}$ (blue) and PL (red) time traces for a CdSe/CdS NC. (C) Histogram of the exciton relaxation times. Red, blue and green histograms correspond to relaxation times extracted from the fits to individual time traces of three different, single NCs. Black histogram shows occurences of relaxation times extracted from averaged traces from NC clusters. (D) Histograms showing the relative contributions of the SE (blue) and the $\Delta$ PL (red) to the total detected signal change $S_{\text {mod }}$ and $P L_{t-}-P_{t+}$, respectively.

We quantified the observed dynamics by simultaneously fitting the PL and $\mathrm{S}_{\text {mod }}$ traces (Supp. Text 5). PL and $S_{\text {mod }}$ traces acquired on small NC clusters revealed that the average slower charge relaxation time is $550 \mathrm{fs}$ (black histogram in Fig. 3C). The time delay traces recorded repeatedly on the same individual NCs (more traces in Fig. S5) revealed the relaxation heterogeneity among individual NCs (Fig. 3C). From the difference in the dynamics between SE and GSD we determined the relative contribution of the two signals to the total measured signal $S_{\text {mod }}$, by performing simple, qualitative kinetic rate equation calculations (Supp. Text 6). The experimental ratio of SE/S $S_{\text {mod }}$ extracted from individual time traces for a large number of NCs centers around a value of $\sim 0.17$ (Fig. 3D). The observation of a ratio $\mathrm{SE} / \mathrm{S}_{\text {mod }}<0.2$ strongly suggests that the cross-sections for absorption and SE might be somewhat different given the large asymmetry between the shape of the absorption and emission bands.

The lower SE signal with respect to GSD signal might also be caused by an excited state absorption (ESA) process. In ESA, the probe beam promotes the excited charges to higher excited states at the cost of absorbing a probe beam photon, leading to a reduction of the apparent SE contrast, enhanced bleaching $(14,20)$ and quenching $(21)$. To uncover the role of ESA in our NC dynamics, we 
varied the duration of the probe pulse, as the ESA timing should be sensitive to the observed 550 fs relaxation time of the hot state. Once the charges have again returned to the emitting state, the probe pulse should stimulate the NC down. The concept, depicted in Fig. 4B, is analoguous to STED experiments, where the STED pulse is stretched to prevent re-excitation (22). We measured the $S_{\bmod }$ and $\Delta \mathrm{PL}$ contrast for increasing probe pulse duration $(\Gamma)$, stretched up to $2.5 \mathrm{ps}$, at $\Delta \mathrm{t}=7 \mathrm{ps}$ delay. In Fig. $4 \mathrm{C}$ both $\mathrm{S}_{\mathrm{mod}}$ and $\Delta \mathrm{PL}$ show increased contrast with the probe pulse duration. Interestingly, the in-growth matches very well the 550 fs excited state charge relaxation time determined from the pump-probe traces. A simulation using the kinetic rate equation model expanded with the ESA process (Supp. Text 7) reproduces the experimental data well and confirms our hypothesis that stretching the stimulating probe pulse allows to stimulate down charges that otherwise undergo ESA.

A

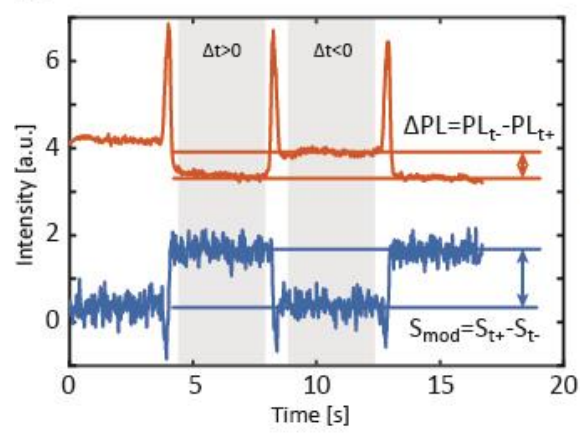

B

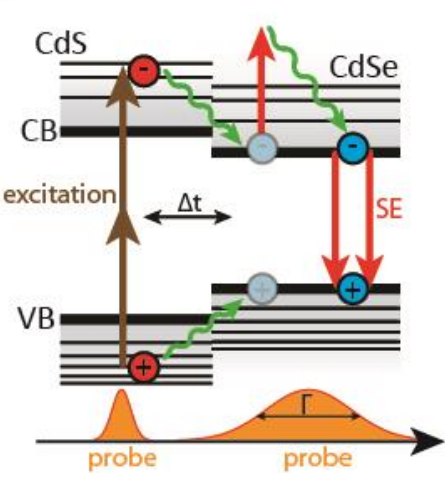

C

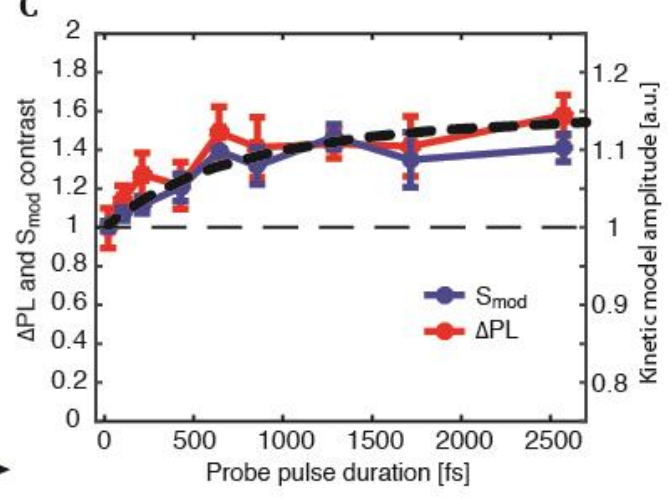

Fig. 4. Higher stimulated emission and photo-luminescence contrast with longer probe pulse. (A) PL and $S_{\text {mod }}$ signals recorded in time while repeatedly scanning the interpulse delay time $\Delta t$ from negative to positive values. (B) Concept of the varying probe pulse duration experiment. (C) Normalized $S_{\bmod }$ and $\Delta \mathrm{PL}$ as a function of probe pulse duration. The traces were averaged from 7 (4 positively and 3 negatively chirped probe traces) separate measurements on different NC clusters. Error bars indicate the standard deviation. Black dashed line is the result of solving the set of kinetic rate equations described in Supp. Text 7.

Interestingly, the simultaneous detection of stimulated and spontaneous emission of a single NC allows us to correlate the decays in quantitative manner. The number of photons detected in SE should be equal to the number of photons missing in PL, that is PL depletion. For the data shown in Fig. 3B, we determined an effective number of photons depleted from PL, $\# \Delta P L_{e f f}=1.6 \cdot 10^{7}$ photons $/ \mathrm{s}$ and an effective number of photons gained in the stimulation beam $\# S E_{e f f}=1.3 \cdot 10^{7}$ photons/s per NC (Supp. Text 8). The two values are in good agreement, given the fact that the detection occurs in two independent channels, using photon counting vs analogue detectors.

The high sensitivity of the presented SE detection opens up new imaging possibilities of weakly fluorescing or quenched systems, while the time-resolved experiment provides information on the excited state relaxation dynamics and its mechanism, all with femtosecond time resolution and singleemitter sensitivity. The unconventional, simultaneous detection of the spontaneous and stimulated emission provides large imaging specificity: the fact that SE depends on two distinct frequencies, in combination with the inter-pulse time-delay, makes the method extremely sensitive to different species within a dense ensemble.

The time-resolved femtosecond SE experiment allowed to us to provide a comprehensive picture of the excited charges, which are either stimulated down, or promoted to higher excited states, or recombine spontaneously. The SE and GSD contributions comprise $<20 \%$ and $>80 \%$ of the total induced ground/excited state population difference, respectively. This was aided by the fact that the two excited charges, electrons and holes, exhibit different relaxation times (Supp. Text 9). The rod-in-rod CdSe/CdS NCs excited holes localize at the core band edge within $200 \mathrm{fs}$, while the excited electrons relax to the core band edge on a time scale of $550 \mathrm{fs}$. We found that the electron relaxation time differs nearly a factor of two between individual NCs. Finally, the single-emitter sensitivity of our experiment allowed us to compare the number of photons lost in PL and gained through SE in absolute terms, which is difficult to 
achieve for ensembles (23). Stretching the stimulation pulse allowed us to elucidate the presence of ESA and increase the SE efficiency by $40 \%-50 \%$, i.e. a significant portion of the excited charges undergo ESA and relax back to the core band edge states.

The ultrafast SE microscopy opens up a spectrum of fascinating experiments to be explored (Supp. Text 10). Scanning the stimulation pulse energy would allow for state selectivity and enable studying excited state-to-state dynamics (16). Given its coherent nature, SE microscopy could be expanded to accommodate heterodyne detection of the stimulation beam and could provide an easy access to investigating coherent effects such as coherent energy transfer $(3,24)$. The absorption crosssection of our NCs at the stimulation wavelength is approximately an order of magnitude larger than the absorption cross-section of a typical fluorescent dye $\left(3 \cdot 10^{-16} \mathrm{~cm}^{2} \mathrm{vs} 10^{-17} \mathrm{~cm}^{2}\right)(25)$. Therefore, potentially even single molecules could be detected in stimulated emission.

\section{References and Notes}

1. S. Yampolsky et al., Nat. Photon. 8, 650-656 (2014)

2. $\quad$ M. Liebel, C. Toninelli, N. F. van Hulst, Nat. Photon. 12, 45-49 (2018)

3. R. Hildner et al., Science 340, 1448-1451 (2013)

4. $\quad$ M. Aeschlimann et al., Science 333, 1723-1726 (2011)

5. V. Kravtsov, R. Ulbricht, J. M. Atkin, M. B. Raschke, Nat. Nanotech. 11, 459-464 (2016)

6. A. Gaiduk, M. Yorulmaz, P. V. Ruijgrok, M. Orrit, Science 330, 353-356 (2010)

7. P. Kukura, M. Celebrano, A. Renn, V. Sandoghdar, J. Phys. Chem. Lett. 1, 3323-3327 (2010)

8. $\quad$ S. Chong, W. Min, X. S. Xie, Phys. Chem. Lett. 1, 3316-3322 (2010)

9. A. B. Zrimsek et al., Chem. Rev. 117, 7583-7613 (2017)

10. W. Min et al., Nature 461, 1105-1109 (2009)

11. S. Christodoulou et al., Nat. Commun. 6, 7905-7913 (2015)

12. J. Hanne et al., Nat. Commun. 6, 7127-7133 (2015)

13. M. D. Lesoine et al., J. Phys. Chem. C 117, 3662-3667 (2013)

14. S. E. Irvine et al., Angew. Chem. 47, 2685-2688 (2008)

15. See supplementary material

16. P. Kambhampati, J. Phys. Chem. C 115, 22089-22109 (2011)

17. E. Hendry et al., Phys. Rev. Lett. 96, 057408-057412 (2006)

18. S. Brovelli et al., Nano Lett. 14, 486-494 (2014)

19. M. Zavelani-Rossi et al., Nano Lett. 10, 3142-3150 (2010)

20. J.-I. Hotta et al., J. Am. Chem. Soc. 132, 5021-5023 (2010)

21. T. Watanabe et al., Chem. Phys. Lett. 420, 410-415 (2006)

22. T. A. Klar, S. W. Hell, Optics Lett. 24, 954-956 (1999)

23. E. Rittweger, B. R. Rankin, V. Westphal, S. W. Hell, Chem. Phys. Lett. 442, 483-487 (2007)

24. A. Chenu, G. D. Scholes, Annu. Rev. Phys. Chem. 66, 69-96 (2015)

25. L. Kastrup, S. W. Hell, Angew. Chem. Int. Ed. 43, 2-5 (2004) 


\section{Acknowledgements}

L.P. acknowledges the Marie Skłodowska-Curie COFUND and the ICFOnest programs. The project has received funding from National Science Centre, Poland, grant 2015/19/P/ST4/03635, POLONEZ 1 and from the European Union's Horizon 2020 research and innovation programme under the Marie Skłodowska-Curie grant agreement No. 665778. This research was funded by the European Commission (ERC Advanced Grant 670949-LightNet), the Spanish Ministry of Economy MINECO (FIS2012-35527, FIS2015-72409-EXP, FIS2015-69258-P, Network FIS2016-81740-REDC 'NanoLight' and Severo Ochoa Grant SEV2015-0522), the Catalan AGAUR (No. 2017SGR1369), Fundació Privada Cellex, Fundació Privada Mir-Puig, and Generalitat de Catalunya through the CERCA Program. Author contributions: L.P. and N.F.v.H. designed the experiment. L.P., N.A. and G.C. performed the experiments and data analysis. S.C. and I.M. provided the samples. L.P. and N.F.v.H. wrote the manuscript. All authors discussed the results and commented on the manuscript. Competing interests: The authors declare no competing financial interests. Data and materials availability: All data are available in the main text or in the supplementary materials (26-36).

\section{Supplementary Materials}

Materials and Methods.

Supplementary Text 1 to 10 .

Supplementary figures S1 to S12.

References 26-36 


\title{
Supplementary Materials for
}

\section{Ultrafast Stimulated Emission Microscopy of Single Nanocrystals}

\author{
Lukasz Piatkowski $^{1 \dagger *}$, Nicolò Accanto ${ }^{1 \ddagger \#}$, Gaëtan Calbris ${ }^{1 \ddagger}$, Sotirios Christodoulou ${ }^{1,2}$ \\ Iwan Moreels 2,3 and Niek F. van Hulst $1,4 *$ \\ ${ }^{1}$ ICFO-Institut de Ciences Fotoniques, the Barcelona Institute of Science and Technology, \\ 08860 Castelldefels (Barcelona), Spain \\ 2 Istituto Italiano di Tecnologia, Via Morego 30, 16163 Genova, Italy \\ 3 Department of Chemistry, Ghent University, Krijgslaan 281-S3, 9000 Gent, Belgium \\ ${ }_{4}^{4}$ ICREA-Institució Catalana de Recerca i Estudis Avançats, 08010 Barcelona, Spain \\ † Present address: Institute of Physical Chemistry, Polish Academy of Sciences, Kasprzaka 44/52, 01-224 \\ Warsaw, Poland \\ \# Present address: Neurophotonics laboratory, Paris Descartes University, 75270 Paris Cedex 06, France. \\ ₹ These authors contributed equally to this work. \\ *E-mail: lukasz.j.piatkowski@put.poznan.pl; Niek.vanHulst@ICFO.eu
}

\section{This file includes:}

Materials and Methods.

Supplementary Text 1 to 10 .

Supplementary Figures S1 to S12.

References 26-36 


\section{Materials and Methods}

\section{Stimulated emission microscopy.}

The experimental setup is schematically depicted in Figure 1A. We used a broadband Ti:sapphire laser in combination with a pulse shaper for dispersion and pulse duration control (26). The Ti:Sapphire laser (Octavius-85M, Thorlabs) was operating at $85 \mathrm{MHz}$ and tuned to a central wavelength of $\sim 760 \mathrm{~nm}$ with a bandwidth of $\sim 200 \mathrm{~nm}$. The outgoing laser beam was spectrally split into an excitation $(\sim 720-850 \mathrm{~nm})$ and stimulation beam $(\sim 650-710 \mathrm{~nm})$. The excitation beam was reflected from a set of dispersion compensating mirrors (Thorlabs) to obtain nearly transform-limited pulses at the sample plane. The stimulation beam was sent through a liquid crystal spatial light modulator (SLM) based, home-built $4 \mathrm{f}$ pulse shaper (adapted from MIIPS-box; Biophotonics Solutions). The pulse shaper was used to compensate for the dispersion and precisely control the pulse duration of the stimulation beam. The stimulation beam was propagated through a delay line (NRT 100/M, Thorlabs), which we used to control the relative time between the excitation and stimulation pulses. The pump beam was transmitted through an acousto-optic modulator (AOM) operating at high ( $\mathrm{MHz}$ ) frequency. After being recombined, the two collinear beams were coupled into an inverted microscope (Observer D1, Zeiss). In the microscope, the broadband pulses were reflected from a 50/50 beam-splitter and focused to a diffraction-limited spot with a high numerical aperture objective (1.3NA, 100x, Zeiss Fluar). The sample was placed on a piezo-controlled stage (Mad City Labs) allowing for precise positioning of the NCs in the focal spot. The photoluminescence from the sample was collected in reflection through the same objective, reflected from a beam-splitter and sent either to a spectrometer equipped with an EM-CCD camera for spectral detection (Newton, Andor) or to an avalanche photodiode (Perkin-Elmer) that allowed confocal imaging of the sample. The photoluminescence was separated from the laser light using two short-pass filters (650SP and 635SP, Semrock). The stimulation beam was collected in transmission with an air objective (0.95NA, 63x, Zeiss Achroplan) and detected with a balanced amplified photodiode (PDB450A, Thorlabs). The pump beam was blocked with a set of $700 \mathrm{~nm}$ short pass filters (Semrock). Modulation depth of the stimulation beam was detected with a lock-in amplifier (Zurich Instruments) locked to the AOM modulation frequency.

\section{Stimulation pulse duration.}

Compression and pulse duration control of the stimulation beam were performed as follows. First, stimulation pulses were compressed to their Fourier limit using the MIIPS method based on $\mathrm{BaTiO}_{3}$ nanoparticles, an approach reported previously (26). Pulse duration of the compressed probe pulses was roughly $25 \mathrm{fs}$, and was verified by measuring the auto-correlation trace and comparing the measured second harmonic spectrum with the simulated one. Then a cross-correlation between the stimulation and pump pulses was measured. The number of reflections for the pump beam of the dispersioncompensating mirror set was adjusted until the cross-correlation trace was shortest. The shortest obtained pump pulse was approximately $50 \mathrm{fs}$.

For tuning the stimulation pulse duration we added positive or negative second order dispersion to the previously acquired compensation mask giving transform limited pulses. We varied the dispersion from $500 \mathrm{fs}^{2}$ to $8000 \mathrm{fs}^{2}$. The relation between the applied chirp and obtained pulse duration is shown in Figure S6A, B. The stimulation pulse duration was determined and confirmed by (i) a simulation using femtoPulse Master (Biophotonic Solutions), (ii) a cross-correlation measurement between the pump and stimulation pulses (Fig. S6C) and (iii) by comparing the acquired second harmonic spectrum with the simulated one for each applied dispersion value (Fig. S6D). The determination of the stimulation pulse duration is very robust and yields small errors. In the experiment, we took great care that the spectral profile and spectrally integrated intensity was the same for probe pulses of different duration. 


\section{Synthesis of CdSe/CdS rods-in-rod NCs.}

For details on the synthesis see reference (11). The dimension of the CdSe core nanocrystals is approximately $4.8 \times 15 \mathrm{~nm}$, and the overall CdSe/CdS diameter and length equaled $9.8 \mathrm{~nm}$ and $44 \mathrm{~nm}$, respectively. The particles were purified by repeated precipitation, centrifugation and resuspension and finally dispersed in chloroform.

\section{Sample preparation.}

The samples were prepared by spin-coating a solution of NCs in PMMA/toluene $(\sim 1 \% \mathrm{w} / \mathrm{v})$ onto a microscope coverslip. The NC concentration and spin-coating parameters were adjusted such to provide NCs at a density of $\leq 0.1$ per $\mu \mathrm{m}^{2}$.

\section{Photon budget calculation.}

Simultaneous detection of the PL and SE allowed us to correlate PL depletion and SE contrast in absolute terms. We compared whether the number of photons lost in PL corresponds to the number of photons gained in the stimulation beam.

The number of photons lost in the PL signal $\triangle P L_{e f f}$ is given by:

$$
\Delta P L_{e f f}=\frac{\Delta P L}{Q E_{N C} \Delta \lambda C_{o b j .} T_{o b j .} T_{B S} Q E_{A P D}}[\# / s]
$$

where $\triangle P L$ is the measured change in the photon count between positive and negative $\Delta t$ in a given PL acquisition timebin. $Q E_{N C}$ is the NC quantum efficiency, given by $Q E_{N C}=Q E_{\text {solution }} \frac{\tau_{\text {single }}}{\tau_{\text {solution }}}=\sim 0.4 \%$, where $Q E_{\text {solution }}=10-15 \%, \tau_{\text {single }}=15-20 \mathrm{~ns}, \tau_{\text {solution }}=700-750 \mathrm{~ns} . \Delta \lambda$ is the ratio of the integrated emission spectrum bandwidth with respect to the total integrated emission spectrum of the $\mathrm{NC}$ and amounts to $\Delta \lambda=1 / 20$. The calculated coupling efficiency of light emitted by an in-plane dipole sitting in the sample plane $C_{o b j}=0.77$. The transmission of the objective given by the manufacturer $T_{o b j .}=0.85$. The measured transmission of the beam splitter $T_{B S}=0.5$ and finally the quantum efficieny of the APD at the specific detection wavelength, specified by the manufacturer, equals $Q E_{A P D}=0.67$. The term $C_{o b j .} T_{o b j .} T_{B S} Q E_{A P D}$ describes the effective detection efficiency of our setup and amounts to about $20 \%$.

The number of extra photons detected in the stimulation beam is defined as:

$$
\Delta S E_{\text {eff }}=\frac{\Delta S E}{2 E_{\text {phot }} R_{P D}} \frac{1}{D_{\text {eff }}}\left[\frac{V W}{s W V}=\# / s\right]
$$

where $\triangle S E$ is the contrast detected with the lock-in amplifier. The measured SE contrast does not depend on the acqusition timebin, but it needs to be included in order to directly compare it to the PL, which depends on the aqusition time interval. The average photon energy of the stimulation pulse is $E_{\text {phot }}=2.94 \cdot 10^{-19} \mathrm{~J}$. The responsivity of the photodiode is $R_{P D}=0.55$ multiplied by the used gain (typically $R_{P D}=0.55 \cdot 10^{6}[\mathrm{~V} / \mathrm{W}]$ ). The term $D_{\text {eff }}$ is the effective detection efficieny of the stimulation beam and was determined experimentaly by comparing the stimulation beam power on top of the sample to the power measured before the photodiode. It thus includes collection efficiency of the upper objective, transmission of the objective $(\sim 85 \%)$, transmission of the fiber $(\sim 93 \%)$ and transmission bandwidth of the short pass filter used for blocking the pump beam. For the 0.95NA objective used in the studies and the two 700SP filters $D_{\text {eff }}=0.8$, meaning that approximately $80 \%$ of the probe beam leaving the sample is detected by the photodiode. 


\title{
Supplementary Text 1 to 10.
}

\author{
Supplementary Text 1: Sign of the lock-in signal \\ We performed additional tests confirming that the detected lockin signal $S_{\text {mod. }}$ indeed has positive sign \\ by measuring intensity of the probe beam transmitted through a dense NC sample with and without the \\ presence of the pump beam (Fig. S7A). Unblocking the pump beam leads to an overal increase in the \\ detected probe beam intensity. Furthermore, the detected signal vanishes when the probe or pump beam \\ is blocked (Fig. S7B). Last but not least, the detected signal is independent of the modulation frequency \\ (Fig. S7C), meaning that we can rule out a contribution from thermal effects, for which the signal typically \\ increases with decreasing modulation frequency (27).
}

\section{Supplementary Text 2: Competition between GSD, SE and PL signals}

Both GSD and SE signals depend on the excited state population and thus compete with the PL. Setting inter-pulse delay too high might lead to lower GSD and $S_{\text {mod }}$ signals, as part of the excited state population would already decay through spontaneous emission. For the used NCs, however, PL occurs on a nanosecond timescale. Therefore on a timescale of few picoseconds the excited state population is hardly affected by PL. The right delay between the excitation and stimulation pulses to ensure efficient interaction with the excited state population is relevant for chromophores for which the excited state lifetime is short (picosecond range) (10).

\section{Supplementary Text 3: Excitation of shell vs core states}

While two-photon absorption involving core-to-core (i.e. CdSe) transitions, including the ones involving the highest valence or lowest conduction band states cannot be explicitly ruled out, we can assume that the two-photon absorption takes place predominantly in the shell region, based on the following arguments:

First, considering the CdSe core and overall CdSe/CdS volumes, we obtain a shell-to-core volume ratio of 11:1. As absorption takes place high above the respective CdSe and CdS band edges, the density of states scales with the volume of the particle. This results in a strongly suppressed absorption in the core compared to absorption in the shell. The linear absorption spectrum of the sample (see Figure 1B of the main text) also confirms this: the vanishing absorption below the CdS edge at $\sim 500 \mathrm{~nm}$ indicates the lower absorption in the CdSe region.

Second, considering that data support a linear absorption involving, at $400 \mathrm{~nm}$, predominantly the shell states, we assume that the two-photon absorption follows the same trend. Indeed, it obeys different selection rules, yet previous results obtained by Allione et al. (28) for similar CdSe/CdS dot-inrod nanocrystals demonstrated that, around $400 \mathrm{~nm}$, both linear and two-photon absorption spectrum are dominated by shell-to-shell transitions. Considering the similarity between both systems, we can extrapolate those results to the current rod-in-rod nanocrystals and conclude that also here, the twophoton absorption around $400 \mathrm{~nm}$ involves predominantly shell-to-shell transitions.

\section{Supplementary Text 4: GSD and SE signatures in the PL trace}

Photoluminescence is a time-integrated signal dependent on the total excited state population generated by both pump and probe pulses. GSD will manifest itself in the photoluminescence trace only if the absorption of the probe pulse (hence population it generates) will differ depending which pulse, pump or probe, came first. If the population generated by each of the two pulses separately is significantly different, then the order, which pulse comes first, which second, is important. In the other extreme, where the two pulses generate exactly the same excited state population, the order of the two pulses does not matter and the total excited state population is exactly the same independent of the interaction order of the two pulses with the sample. In our experiment, lower two-photon excitation probability with the pump pulse (with respect to a single photon excitation probability with the probe pulse) is compensated by higher absorption cross-section in the $400 \mathrm{~nm}$ range. Consequently the pump and probe pulses generate nearly the same excited state population, as evident in the PL time trace shown in the Fig. S7C. It is therefore reasonable that in the acquired PL time traces we are sensitive exclusively to the stimulated emission process. 


\section{Supplementary Text 5: Fitting the $S_{\text {mod }}$ and PL dynamics}

To quantify the observed dynamics we simultaneously fitted the PL and $S_{\bmod }$ traces to an exponential function with an offset in the form $a+b \cdot e^{-t / \tau}$. In the fit we kept the longer relaxation time parameter $\tau$ the same for both traces. The amplitudes $a_{s}, b_{S}$ and $a_{P L}, b_{P L}$ were used as independent fit parameters for $S_{\text {mod }}$ and PL traces, respectively. All fits were done starting from $t=250$ fs to avoid any contribution of the pump and probe pulse cross-correlation signal (FWHM of $\leq 200 \mathrm{fs}$ ). Consequently, as is the total amplitude of the $S_{\text {mod }}$ Signal, $\left(a_{S}-b_{S}\right)$ parameter describes the contribution of the GSD to the $S_{\text {mod }}$ signal, whereas $b_{S}$ corresponds to SE contribution. Respectively, parameter $b_{\mathrm{PL}}$ reflects the amplitude of the photoluminescence depletion $\triangle \mathrm{PL}$. PL and $\mathrm{S}_{\text {mod }}$ traces acquired on small NC clusters revealed that the average slower charge relaxation time is $550 \mathrm{fs}$ (black histogram in Fig. $3 \mathrm{C}$ ). The delay time-dependent traces recorded repeatedly on the same individual NCs demonstrated relaxation heterogeneity among individual NCs. Additional interpulse delay time dependent traces for NC clusters and individual NCs are shown in the Figure S5.

\section{Supplementary Text 6: Kinetic rate equation modelling}

Here, one should consider what $S_{\text {mod }}$ represents. Both SE and GSD are directly proportional to the excited state population $\left(\mathrm{N}_{1}\right)$. If we assume here that: (a) since the excited state population relaxes very fast (subpicosecond timescale), no significant charge trapping occurs and all the population excited by the pump beam relaxes to the core band edge, and (b) the cross-sections for SE ( $\left.\sigma_{\mathrm{SE}}\right)$ and absorption of the probe pulse $\left(\sigma_{a b s}\right)$ are the same, then the GSD and SE response are equal, because they report on exactly the same population - GSD from the perspective of the ground state (GSD $\propto \sigma_{a b s} \cdot N_{1}$, where $N_{1}=N_{0}-\Delta N$ ) and $\mathrm{SE}$ from the perspective of the excited state $\left(\mathrm{SE} \propto \sigma_{\mathrm{SE}} \cdot \mathrm{N}_{1}\right)$. Consequently the ratio of $\mathrm{SE} / \mathrm{S}_{\text {mod }}$ should be equal to 0.5 . The experimental ratio of SE/ $S_{\text {mod }}$ extracted from individual time traces for a large number of NCs centers around a value of $\sim 0.17$ (Fig. 3D). The observation of a ratio SE $/ \mathrm{S}_{\text {mod }}<0.2$ strongly suggests that the assumption of equal cross-sections is invalid.

In order to understand how does the $S_{\text {mod }}$ signal behave in time and what is the relative contribution of the GSD and SE to $S_{\text {mod }}$ we performed simple kinetic rate equation modelling. We used an intuitive approximation of the experiment using three level system shown in Supp. Fig. S8. Brown and red arrows indicate all possible transitions caused by the pump and probe pulses, respectively. Green arrow indicates internal relaxation which reflects charge relaxation to the lowest excited core state. Purple arrow corresponds to the spontaneous emission. Black dotted arrow indicates an excited state population loss channel that simulates plausible charge trapping by the surface and shell/core interface defects. In this model for simplicity we assume that one of the charges relaxes immediately to the lowest excited state and that the GSD develops with the excitation pulse duration.

Kinetic rate equations describing temporal evolution of the population of each state take form:

$$
\begin{aligned}
& \frac{d N_{0}}{d t}=- \text { Pulse }_{\text {pump }}(t) N_{0}+\text { Puls } e_{\text {pump }}(t) N_{2}-\text { Pulse }_{\text {probe }}(t) N_{0}+\text { Pulse }_{\text {probe }}(t) N_{1}+k_{\text {emm. }} N_{1} \\
& \frac{d N_{1}}{d t}=- \text { Pulse }_{\text {probe }}(t) N_{1}+\text { Pulse } e_{\text {probe }}(t) N_{0}+k_{\text {rel. }} N_{2}-k_{\text {emm. }} N_{1} \\
& \frac{d N_{2}}{d t}=\text { Pulse }_{\text {pump }}(t) N_{0}-\text { Pulse } e_{\text {pump }}(t) N_{2}-k_{\text {rel. }} N_{2}-\left(k_{\text {dark }} N_{2}\right)
\end{aligned}
$$

For the pump and probe pulses we used Gaussian function: Pulse $=k_{\text {exc@peak }} e^{-(t-\Delta t)^{2} / 2 c^{2}}$, where the excitation rate peak is given by $k_{\text {exc@peak }}=\frac{\sigma_{\text {abs }} I_{\text {peak }} \lambda}{h c}$ and $I_{\text {peak }}=\frac{P_{\text {ave }} \text { focus }_{\text {area }}}{\text { repetition }_{\text {rate }} \text { pulse }_{\text {duration }}}$.

In order to simulate the detected $S_{\text {mod }}$ signal we solved the set of kinetic rate equations twice: once including the excitation by the pump pulse and once setting Pulse $e_{\text {pump }}$ to zero, emulating the modulation of the pump beam in the experiment. Both calculations were done for different $\Delta t$ delay times between the pump and probe beams ( $\Delta t=-1$ to $+7 \mathrm{ps})$. 
$\mathrm{S}_{\text {mod }}$ signal only involves interaction between the sample and the probe pulse. Therefore, we only take into account changes to the probe intensity caused by the interaction of the probe with the ground state population $N_{0}$ (absorption $P_{\text {probe }}(t) N_{0}$ ) and excited state population $N_{1}$ (stimulated emission $P_{\text {probe }}(t) N_{1}$ ). Finally, $S_{\text {mod }}$ signal is proportional to the difference in the change of the probe beam intensity when passing through the sample that was excited with the change of the probe beam intensity passing through the sample that was not excited:

$$
\begin{aligned}
\mathrm{S}_{\text {mod }}(\Delta \mathrm{t})=\Delta I_{\text {probe }}(\Delta t) & =\left[P_{\text {probe }}(t) N_{1}-P_{\text {probe }}(t) N_{0}\right]_{\text {with pump }} \\
& -\left[P_{\text {probe }}(t) N_{1}-P_{\text {probe }}(t) N_{0}\right]_{\text {no pump }}
\end{aligned}
$$

The simulated $S_{\text {mod }}(\Delta t)$ is shown in Figure S8B with blue circles. We further treated this simulated data like the experimental data, that is starting from $\Delta t=250$ fs we fitted an exponential function $a+$ $b \cdot e^{-t / \tau}$ and determined the ratio SE/S $S_{\text {mod. }}$ Fitted curves are marked with read lines in Fig. S8B. The ratio $\mathrm{SE} / \mathrm{S}_{\text {mod }}$ is plotted in Fig. S8C as a function of fraction of the experimental probe and pump pulse intensity. Fraction 1 corresponds to experimental conditions. We varied the pump and probe intensity by roughly an order of magnitude around the experimental value and found only a small effect on the determined $\mathrm{SE} / \mathrm{S}_{\text {mod }}$ ratio.

Next, we checked the evolution of the $S_{\text {mod }}$ signal assuming that the absorption and stimulated emission cross-sections are not the same. We varied the absorption to stimulated emission (Abs/SE) cross-section ratio from 1 to 4 and found that increasing absorption cross-section relative to SE crosssection leads to a significant decrease of the SE/S $\mathrm{S}_{\text {mod }}$ ratio.

Lifting the second assumption, that all the excited state population relaxes to the lowest excited core state, leads to a similar effect. Here we added a fast relaxation channel to a dark (trap) state $\left(-k_{\text {dark }} N_{2}\right)$ and varied the relaxation time $1 / k_{\text {dark. }}$ The result is shown in Fig. S8E.

We note here that detailed studies of the density and character of states are required to model the charge dynamics and measured quantities in a quantitative manner, which is out of the scope of this manuscript.

\section{Supplementary Text 7: Kinetic rate equation modelling including ESA}

The delay between the pump and probe pulses was kept constant at $\Delta t=7 \mathrm{ps}$. In the calculation we only varied parameter $c$ in Pulse $=k_{\text {exc@peak }} e^{-(t-\Delta t)^{2} / 2 c^{2}}$, such that we obtained a set of 9 probe pulse durations with FWHM between $\Gamma=20$ fs and 2770 fs.

For simulating the dependence of the $S_{\bmod }$ on the probe pulse duration we used the same approach as in Supp. Text 6. We only modified the set of kinetic rate equations by adding additional (ESA) state (marked red). For simplicity we used the following assumptions: (a) all the charges excited to higher excited state $\left(\mathrm{N}_{3}\right)$ return to the lowest excite state $\left(\mathrm{N}_{1}\right)$, (b) the probability (cross-section) for absorption, stimulated emission and excited state absorption are the same, that is Ratio ${ }_{E S A} / S E=1$ and (c) the energy separation between $\mathrm{N}_{2}$ and $\mathrm{N}_{3}$ states is small, hence we take the relaxation rate from $\mathrm{N}_{3}$ to be the same as from $\mathrm{N}_{2}$, denoted as $k_{\text {rel }}$. Regarding assumption (b), theoretical work by Norris et al. showed that comparable transition strengths were found for the first transition and excited state transitions in CdS QDs (29).

$$
\begin{aligned}
\frac{d N_{0}}{d t}= & - \text { Pulse }_{\text {pump }}(t) N_{0}+\text { Puls }_{\text {pump }}(t) N_{2}-\text { Pulse }_{\text {probe }}(t) N_{0}+\text { Pulse }_{\text {probe }}(t) N_{1}+k_{\text {emm. }} N_{1} \\
\frac{d N_{1}}{d t}= & - \text { Pulse }_{\text {probe }}(t) N_{1}+\text { Pulse }_{\text {probe }}(t) N_{0}+k_{\text {rel. }} N_{2}-k_{\text {emm. }} N_{1} \\
& - \text { Ratio }_{E S A / S E} \text { Pulse }_{\text {probe }}(t) N_{1}+k_{\text {rel. }} N_{3} \\
\frac{d N_{2}}{d t}= & \text { Pulse } e_{\text {pump }}(t) N_{0}-\text { Pulse }_{\text {pump }}(t) N_{2}-k_{\text {rel. }} N_{2}-\left(k_{\text {dark }} N_{2}\right) \\
\frac{d N_{3}}{d t}= & \text { Ratio }_{E S A / S E} \text { Pulse }_{\text {probe }}(t) N_{1}-k_{\text {rel. }} N_{3}
\end{aligned}
$$


Such that,

$$
\begin{aligned}
& S_{\text {mod }}(\Gamma)=\Delta I_{\text {probe }}(\Gamma)=\left[\text { Pulse }_{\text {probe }}(t) N_{1}-P_{\text {probe }}(t) N_{0}-\text { Ratio }_{E S A / S E} \text { Pulse }_{\text {probe }}(t) N_{1}\right]_{\text {with pump }} \\
& -\left[\text { Pulse }_{\text {probe }}(t) N_{1}-P_{\text {probe }}(t) N_{0}-\text { Ratio }_{E S A / S E} \text { Pulse }_{\text {probe }}(t) N_{1}\right]_{\text {no pump }}
\end{aligned}
$$

It is important to note here that an in-depth theoretical input regarding the charge trapping efficiencies, the exact excited states involved in the experiment and cross-sections for SE and absorption processes is needed to quantitatively capture the magnitude of the effect.

\section{Supplementary Text 8: Photon budget and saturation conditions}

We compared the number of photons lost in the PL trace with the number of photons gained through SE process. For this calculation we used the data shown in Figure 3B. We note that the antibunching trace for this particular measurement indicates presence of $n=1-2$ NCs (Fig. S3). For these time traces we found $\Delta \mathrm{PL}=900$ counts per second and $\mathrm{SE}=\sim 1.9 \cdot 10^{-7}$. Given the detection efficiencies of both detection channels (for details see Materials and Methods: Photon budget calculation) we found an effective number of photons depleted from PL, $\triangle P L_{e f f}=1.6 \cdot 10^{7}$ per $1 \mathrm{~s}$, and an effective number of photons gained in the stimulation beam $\Delta S E_{\text {eff }}=1.3 \cdot 10^{7}$ per $1 \mathrm{~s}$.

We should note here that for the given laser repetition rate of $8.5 \cdot 10^{7}$ per $1 \mathrm{~s}$, in case of excitation and stimulation saturation conditions maximum obtainable photon count is half of the repetition rate, that is $4.25 \cdot 10^{7}$ per $1 \mathrm{~s}$. We detect $\sim 1.6 \cdot 10^{7}$ photons for at most $2 \mathrm{NCs}$, which means that for a single NC we have at most $0.8 \cdot 10^{7}$ photons per $1 \mathrm{~s}$. This is roughly $35 \%$ of the total detected photoluminescence counts, meaning that we actually detect at most $\sim 2 \cdot 10^{7}$ photons per second per NC. This puts us at about $50 \%$ of the saturation conditions, in agreement with the spectral position of the emission spectrum of the NC (Fig. S1).

Furthermore, GSD is detected within a few hundreds of femtoseconds after excitation, hence it is unlikely to be significantly affected by charge trapping and repopulation of the ground state. This means that GSD reflects the total number of electron-hole pairs generated by the pump beam. The number of detected PL photons resulting from pump-only excitation (probe beam blocked) is directly linked to the number of generated electron-hole pairs through quantum efficiency $\mathrm{QE}_{\mathrm{NC}}: \# P L_{\text {pump }}=\# G S D_{\text {eff }} \cdot Q E_{N C}$. Using the same calculation, we found $\# G S D_{\text {eff }}=0.5 \cdot 10^{7}$ and $\# P L_{\text {pump }}=0.8 \cdot 10^{7}$ excitations $/ \mathrm{s}$ per NC. Moreover, the nice agreement between these numbers confirms the quantum efficiency of the individual NCs (see Materials and Methods).

\section{Supplementary Text 9: Different charge relaxation times in core-shell structures.}

In the relaxation process, prior to intraband relaxation, the carriers first need to transfer from the shell to the core region. However, here we clustered both processes in an overall relaxation to highlight that electron and hole relaxation/transfer dynamics can occur on different timescales.

When looking at the existing literature, we can observe that:

1. Intraband relaxation for electrons occurs via Auger-mediated heating of the hole. This is due to the sparsity of the electron density of states near the band edge, preventing efficient phonon-mediated relaxation. As a consequence, electron relaxation rates are faster than hole relaxation rates, both are however proceeding on a sub-picosecond time scale $(16,17)$. In case of our CdSe/CdS NCs, however, it is more likely that the holes relax faster, because of the smoother potential energy landscape for the holes compared to the electrons (11). Consistently, for similar CdSe/CdS heterostructures it was shown that the specific band edge alignment favors faster hole localization in the core $(18,19,29)$. Furthermore in $\mathrm{CdSe} / \mathrm{CdS}$ dot-in-rod NCs excited hole relaxation was found to occur within a picosecond $(31,32)$.

2. For the shell-to-core transfer rate, a paper by Galland et al. on dot-in-bulk CdSe/CdS (33), shell-tocore volume ratio up to 185:1), found a typical hole transfer rate of about $10 \mathrm{ps}$. This value decreases 
strongly however for CdSe/CdS dot-in-rod nanocrystals, where Lupo et al. measured a value of $650 \mathrm{fs}$ (31). That values depend on the geometry, was also confirmed by Diroll et al. (34), who used ultrafast fluorescence spectroscopy to investigate core and shell emission in CdSe/CdS dot-in-rod nanocrystals, and measured shell fluorescence decay times up to 1.4 ps (for the fast component). More importantly however, different values were measured for the core fluorescence build-up time, confirming that electron and holes are transferred from shell to core on a different time scale.

In conclusion, we build upon existing literature to conclude that electrons and holes have a different shell-to-core relaxation time, as well as a different intraband relaxation time. Typical decay times for intraband relaxation are below 1 picosecond, while for the transfer times literature values vary over a larger range, but strongly depend on the geometry and shell-to-core volume ratio and can still occur on a time scale of about $500 \mathrm{fs}$. The extracted relaxation times refer to the overall relaxation pathway (from initial highly excited state to lowest excited state) of the charges that undergo a complete photocycle.

\section{Supplementary Text 10: SE microscopy on other systems.}

To demonstrate the applicability of SE microscopy to other systems, below we show simultaneously acquired PL and $\mathrm{S}_{\text {mod }}$ images for three different systems: (a) large rod-in-rod CdSe/CdS nanocrystals, (b) light harvesting complex 2 (LH2) nanocrystals and (c) QDI organic chromophores. It is evident that our approach can be used to both semiconducting and chromophore systems, although it should be noted that particular system of interest requires careful selection of experimental conditions: single vs twophoton excitation and stimulation; spectral and temporal characteristic of pump and probe pulses; spectral and dispersion characteristic of all optical elements - all these are particularly important for quantitative analysis. For CdSe/CdS NCs we used two-photon excitation and single photon stimulation, analogously to the experiment presented in the manuscript, while for LH2 nanocrystals and for QDI chromophores we used single photon excitation and stimulation.

Each of the systems is quite different: semiconducting (CdSe/CdS) vs chromophore (LH2, QDI), and efficient (QDI) vs weak emitter (LH2). They have very different spectral signatures, lifetimes, shape and sizes. Consequently, the here presented data were acquired with different experimental conditions in each case; hence, we focus on demonstrating qualitative, rather than quantitative comparison.
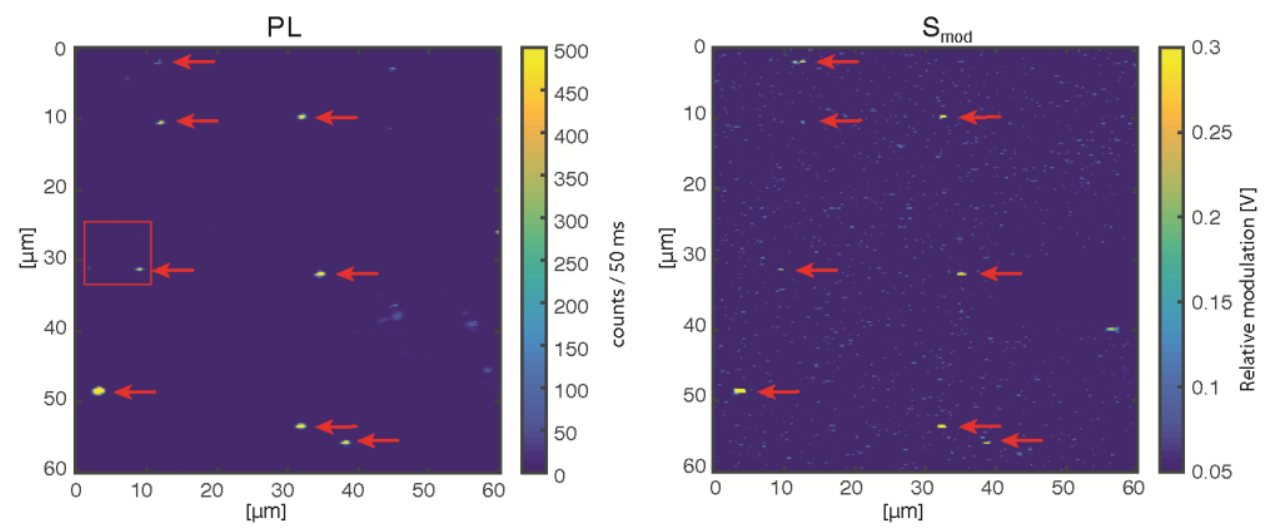

Fig. S9. Simultaneous PL and $S_{\text {mod }}$ imaging of redshifted rod-in-rod CdSe/CdS NCs. These are large $(\sim 30 \mathrm{~nm} x \sim 85 \mathrm{~nm})$ NCs referred to as RIR III in reference (11). 

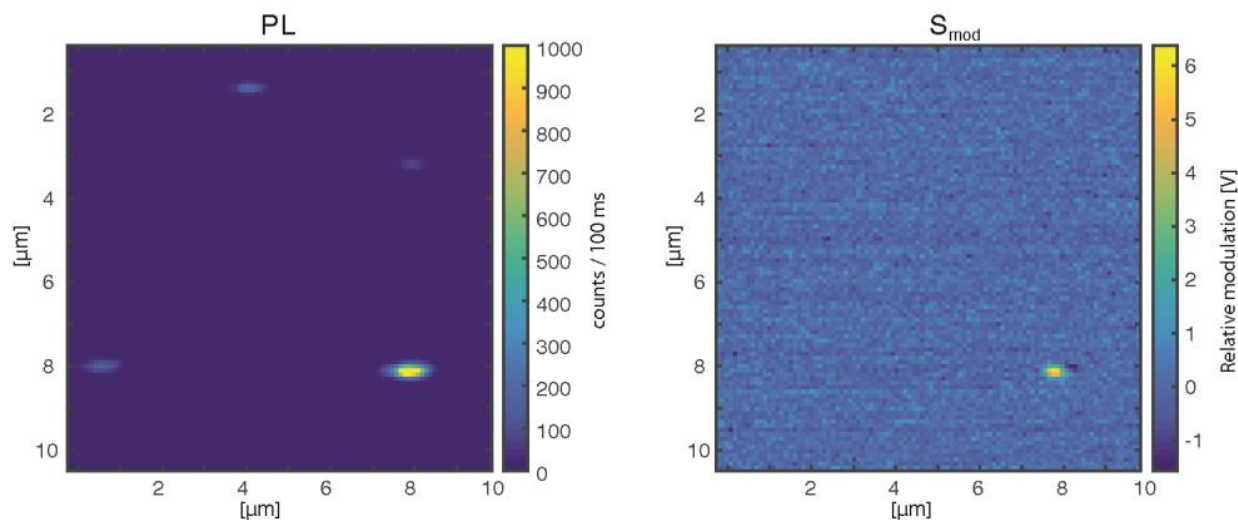

Fig. S10 Simultaneous PL and $S_{\text {mod }}$ imaging of redshifted rod-in-rod CdSe/CdS NCs.

Figure shows a zoomed-in view on the area marked with a red quare in Fig. S9. Estimated absolute modulation depth for the $S_{\bmod }$ signal is $\sim 10^{-5}$.
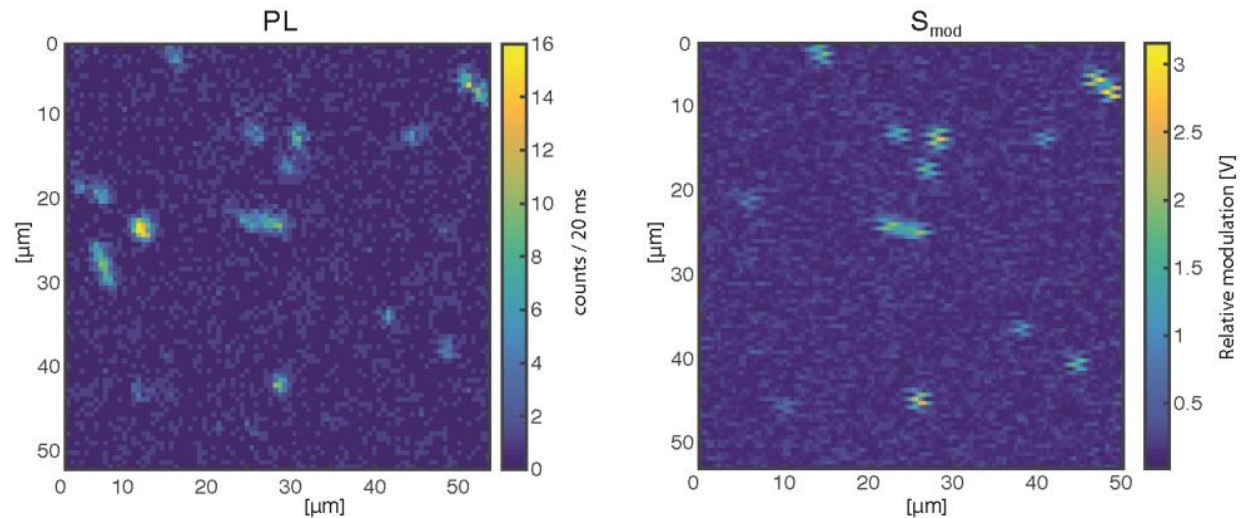

Fig. S11. Simultaneous PL and $\boldsymbol{S}_{\text {mod }}$ imaging of LH2 nanocrystals. The spin-coated LH2 nanocrytals are a few hundred of $n m$ in size (35). Estimated absolute modulation depth for the $S_{\text {mod }}$ signal is $\sim 10^{-5}$.
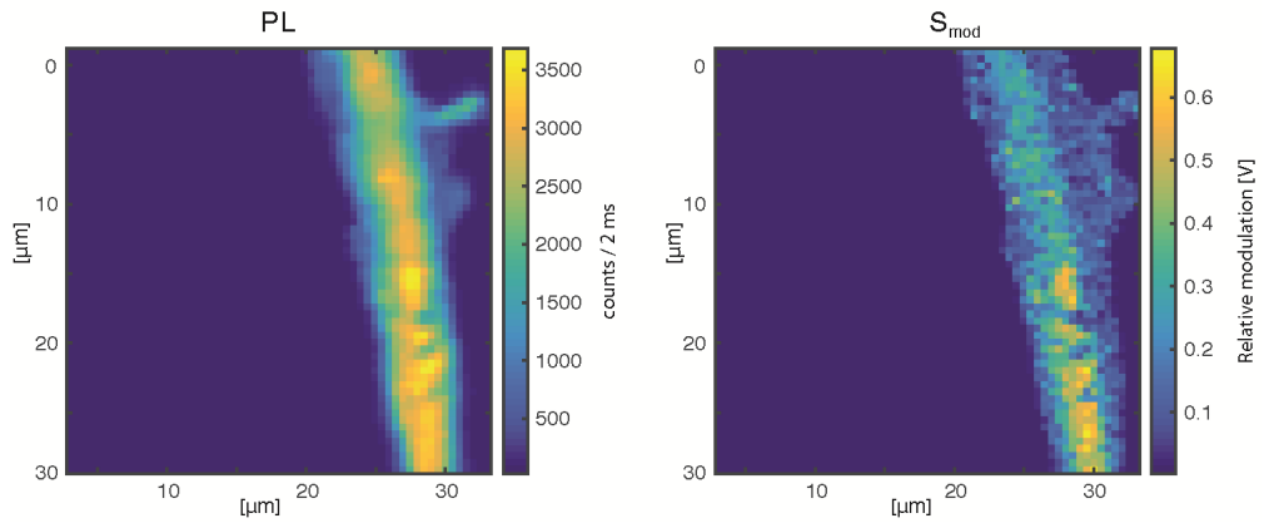

Fig. S12. Simultaneous PL and $S_{\text {mod }}$ imaging of dropcasted QDI chromophores. QDI is an analog of a terrylene dye, typically used in single molecule studies (36). Here we drop-casted $1 \mu \mathrm{M}$ solution onto a glass cover slip and imaged the dried out sample. Estimated absolute modulation depth for the $S_{\text {mod }}$ signal is $\sim 10^{-4}$ 


\section{Supplementary figures S1 to S8}

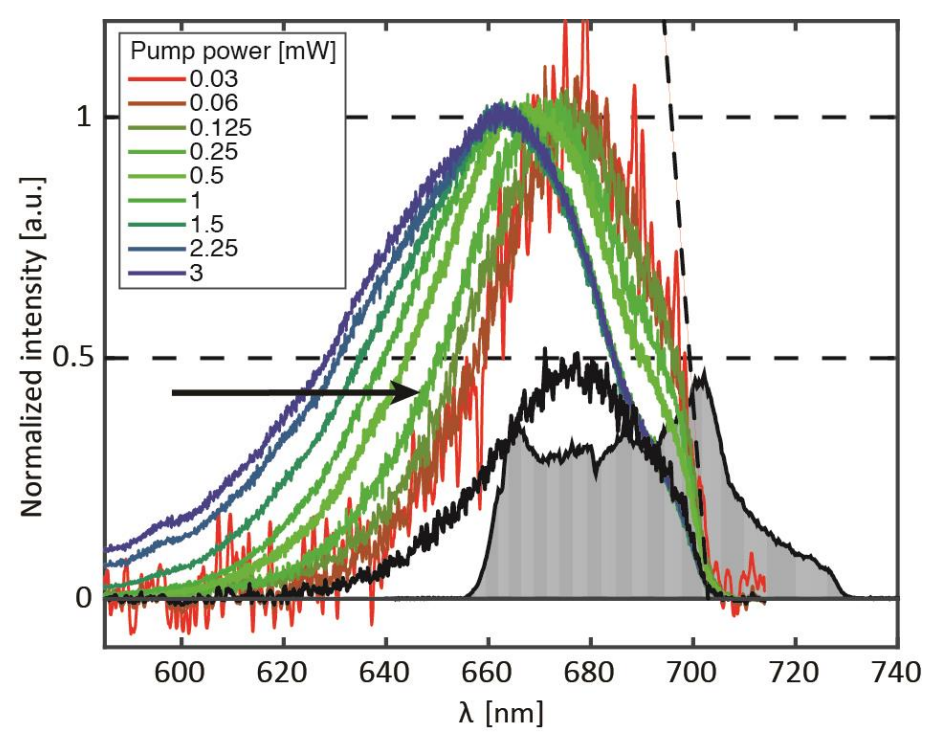

Supplementary Figure S1. PL emission spectra as a function of excitation intensity.

PL emission spectra are given with colored lines. Black spectrum is scaled (x0.5) emission spectrum measured with the excitation power used in the experiments. Grey shaded spectrum is the stimulation pulse spectrum normalized to value of 0.5 . The vertical dashed line indicates the cut-off slope of a short pass filter. For given CdSe/CdS rod-in-rod nanocrystals intense excitation may lead to a generation of multiple charge pairs (multiexcitons). Multiple charges interact with each other leading to a blue shift of the emission spectrum (11). It is thus straightforward to establish whether we operate in a single or multiple charge pair regime, by measuring emission spectra as a function of excitation intensity. Given our experimental conditions ( $85 \mathrm{MHz}$ repetition rate, $800 \mathrm{~nm}$ central pump wavelength, focal spot size; see Materials and Methods) we determined that we operate in the single charge pair regime, close to the onset of multiple charge pair generation. 

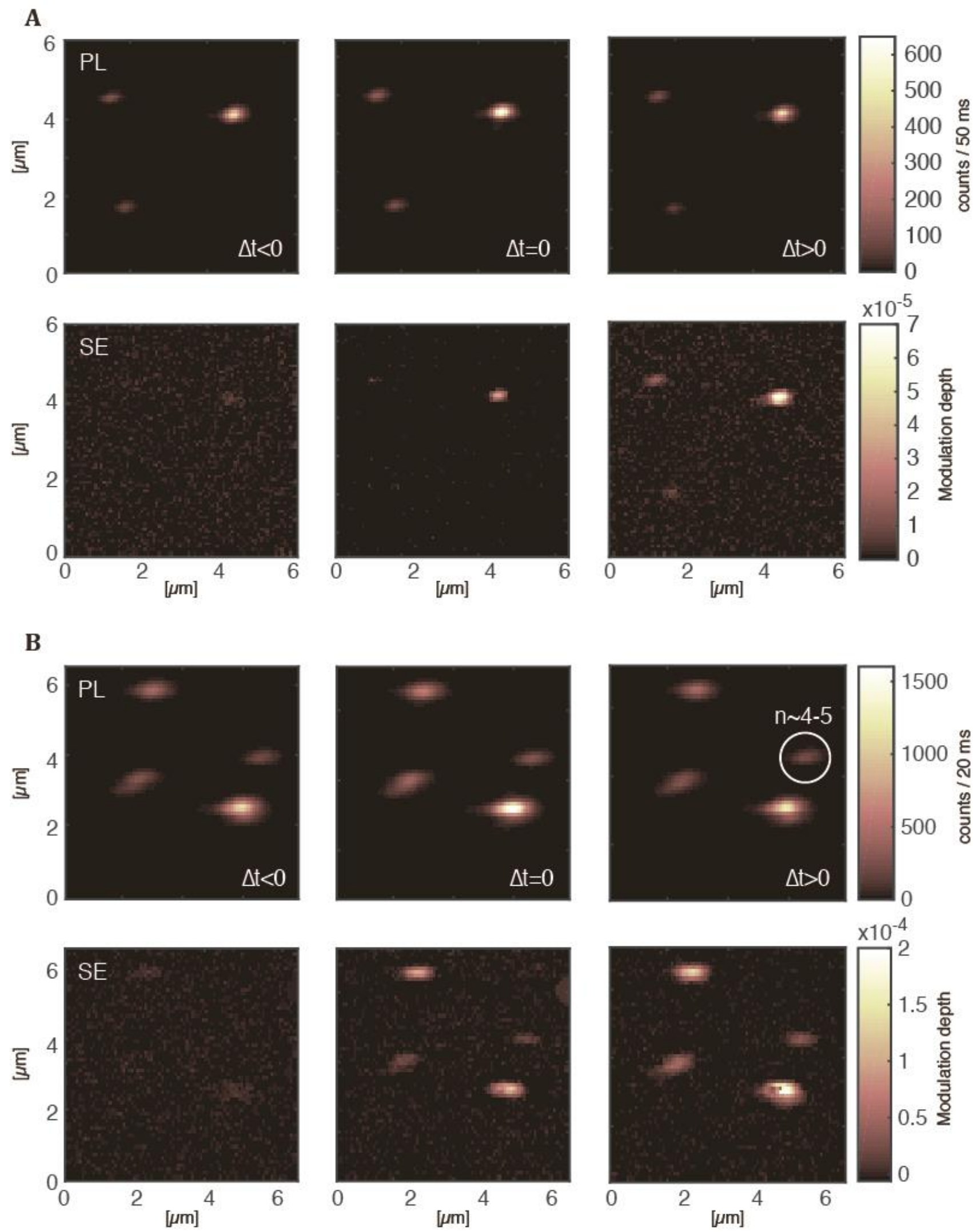

Supplementary Figure S2. Simultaneous SE and PL imaging of NCs.

A series of images of two areas of the sample (A and B) acquired by detecting PL (top row) and SE (bottom row) signals, for different time delays $\Delta t$ between the excitation and stimulation pulses (left to right). 


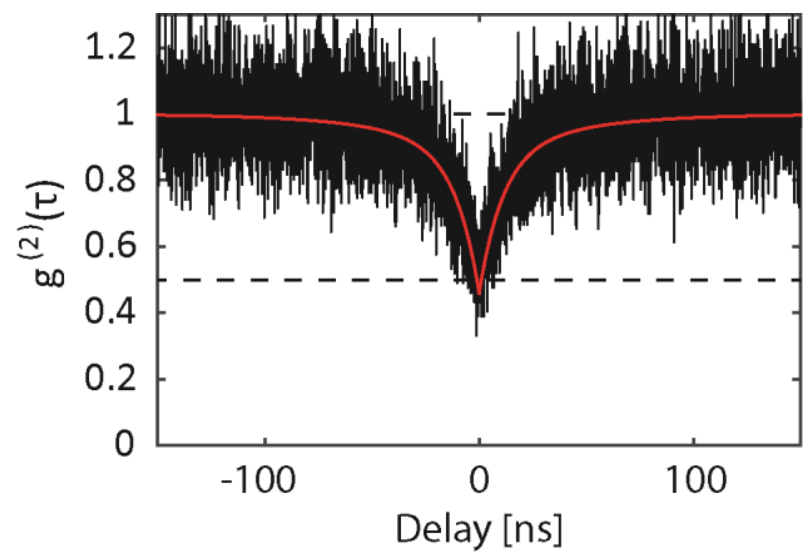

Supplementary Figure S3. Anti-bunching trace.

Photon anti-bunching trace corresponding to the NC denoted $x$ in Figure 3A.

A

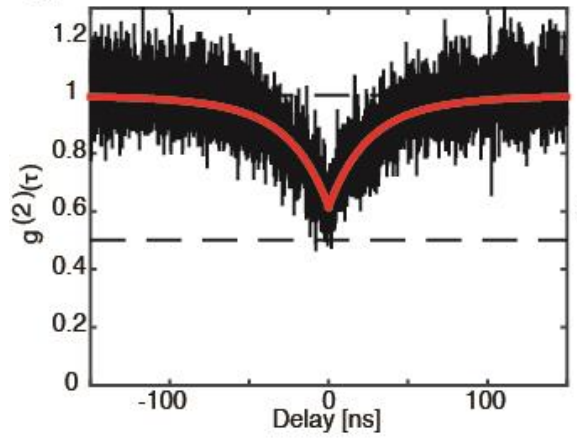

B

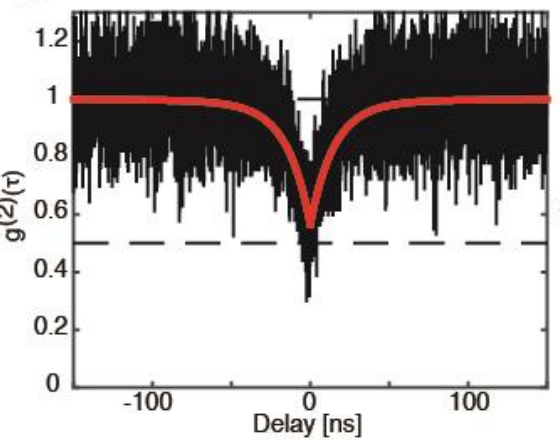

C

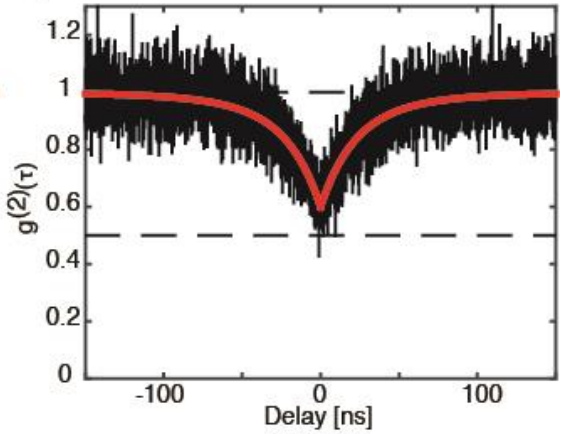

\section{Supplementary Figure S4. Anti-bunching traces.}

Photon anti-bunching traces for NC data shown in Figure 3B and discussed in the main text (B) and (A, C) for NC data shown in Figure S5A and B, respectively. 
A

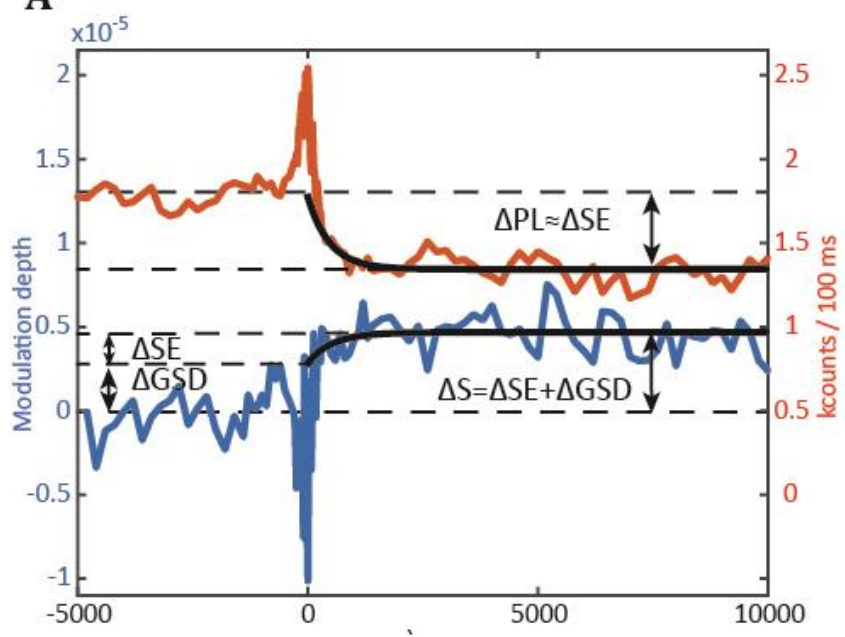

C

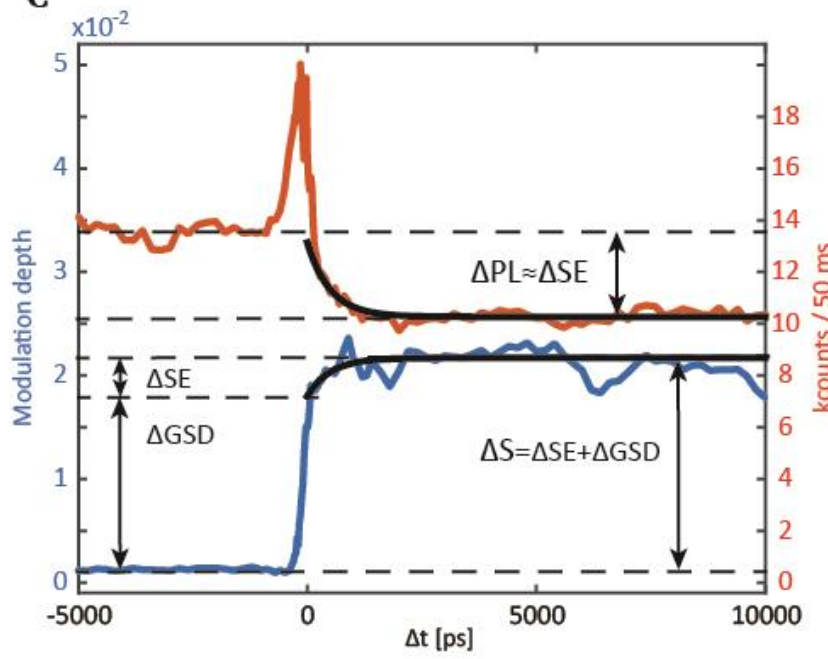

B

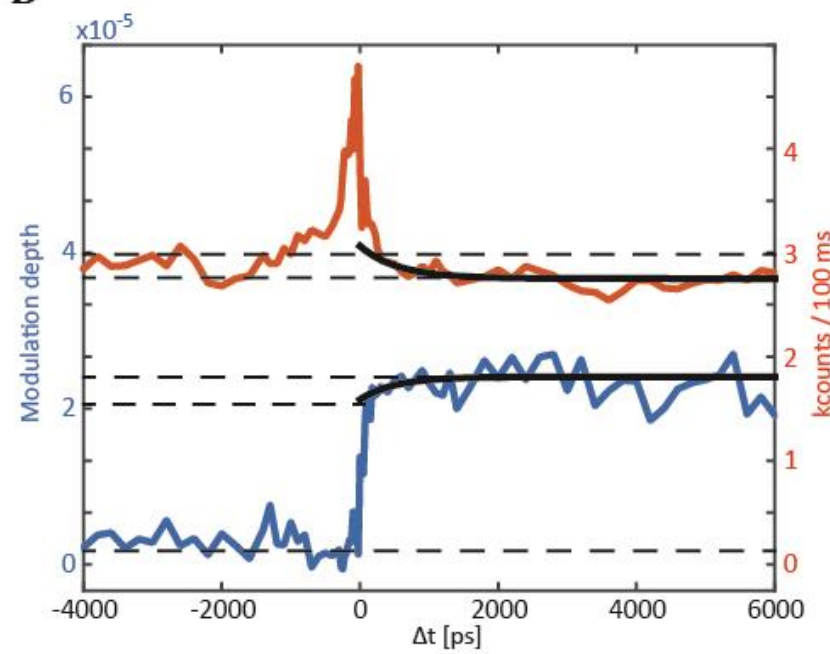

D

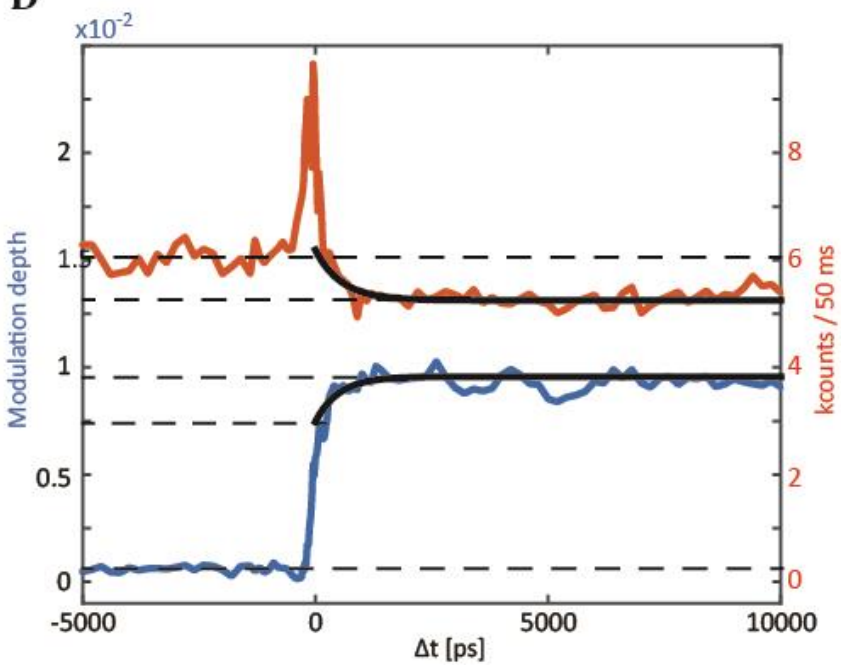

Supplementary Figure S5. SE and PL time traces.

Simultaneously detected SE (blue) and PL (red) time traces for individual NCs (A, B) and NC clusters (C, D). For traces shown in panels $\mathbf{A}$ and $\mathbf{C}$ we indicate the contributions of SE and GSD to the measured $\Delta \mathrm{SE}$ and $\triangle \mathrm{PL}$ signals. Antibunching traces corresponding to NCs shown in panels $\mathbf{A}$ and $\mathbf{B}$ are shown in Figure S4. 

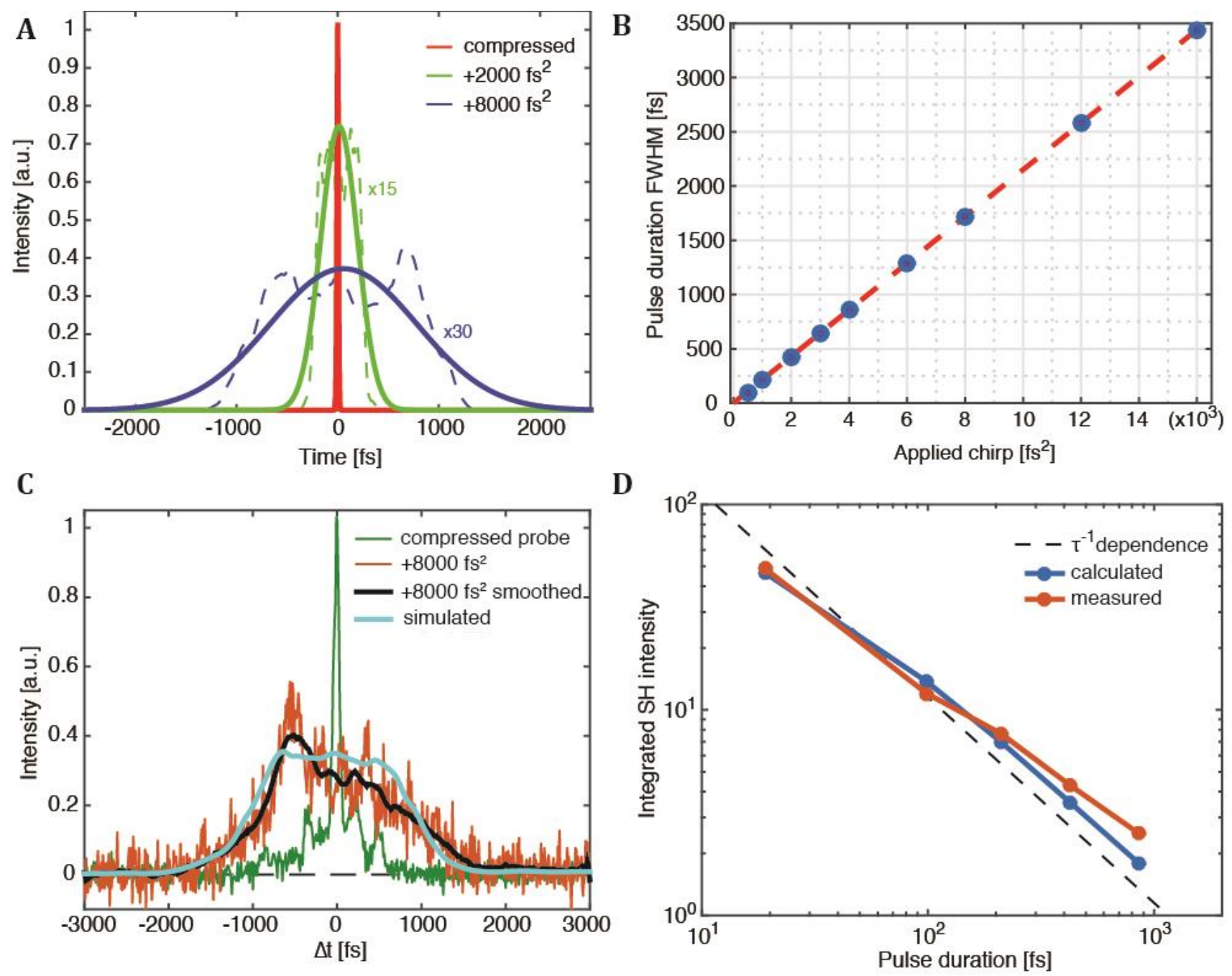

\section{Supplementary Figure S6. Probe pulse characteristic as a function of pulse duration.}

(A) Dashed lines indicate calculated temporal profiles of compressed probe pulse (red) and upon addition of quadratic chirp of different values (green and blue). The temporal profiles are calculated based on the probe pulse spectral intensity and applied dispersion compensation mask, using femtoPulse Master software (Biophotonic Solutions). Solid lines indicate corresponding Gaussian fits. (B) Points indicate probe pulse durations for a given chirp applied to the SLM. Dashed line acts as guide to the eye. (C) Experimental cross-correlation traces between the compressed pump pulse and compressed and stretched probe pulse indicated with green and red lines, respectively. Pump pulses were compressed by reflecting them from a set of dispersion compensating mirrors. Probe pulses were compressed using SLM-based pulse shaper. Light blue line indicates calculated temporal profile of the probe pulse with $+8000 \mathrm{fs}^{2}$ chirp applied to the SLM. Good correspondence is found between the experimental cross-correlation trace and calculated stretched probe pulse temporal profile. (D) Points indicate spectrally integrated intensities of the measured (red) and calculated (blue) second harmonic signal generated with a probe pulse from a $\mathrm{BaTiO}_{3}$ nanoparticles (26). Dashed line indicates the typical 1 /pulse duration dependence of the second harmonic signal. 
A

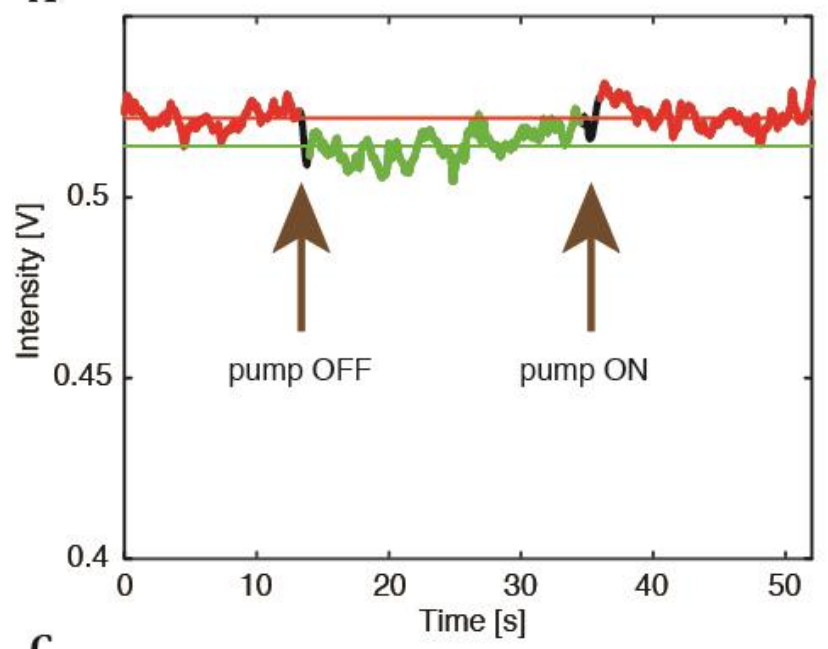

B

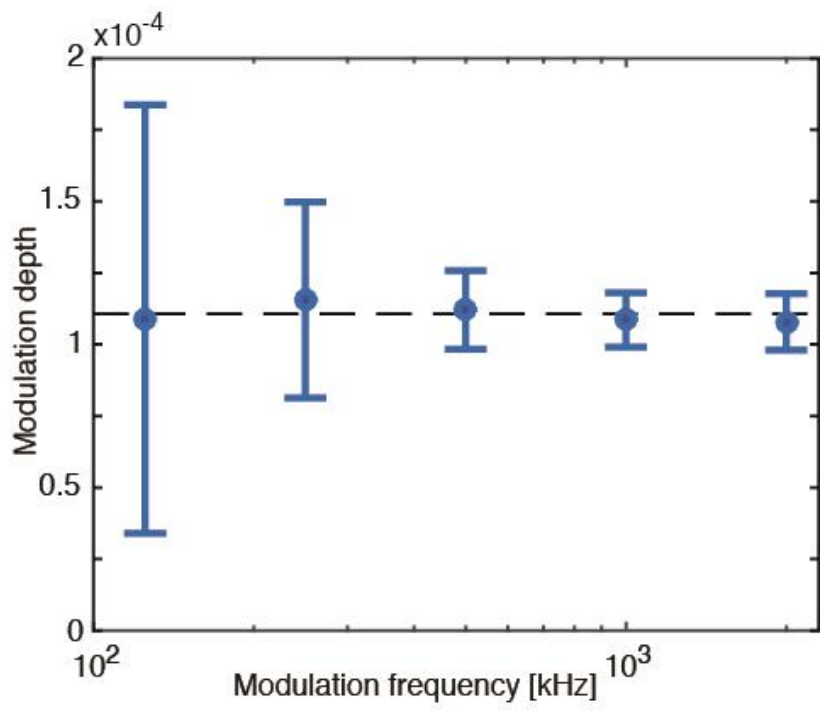

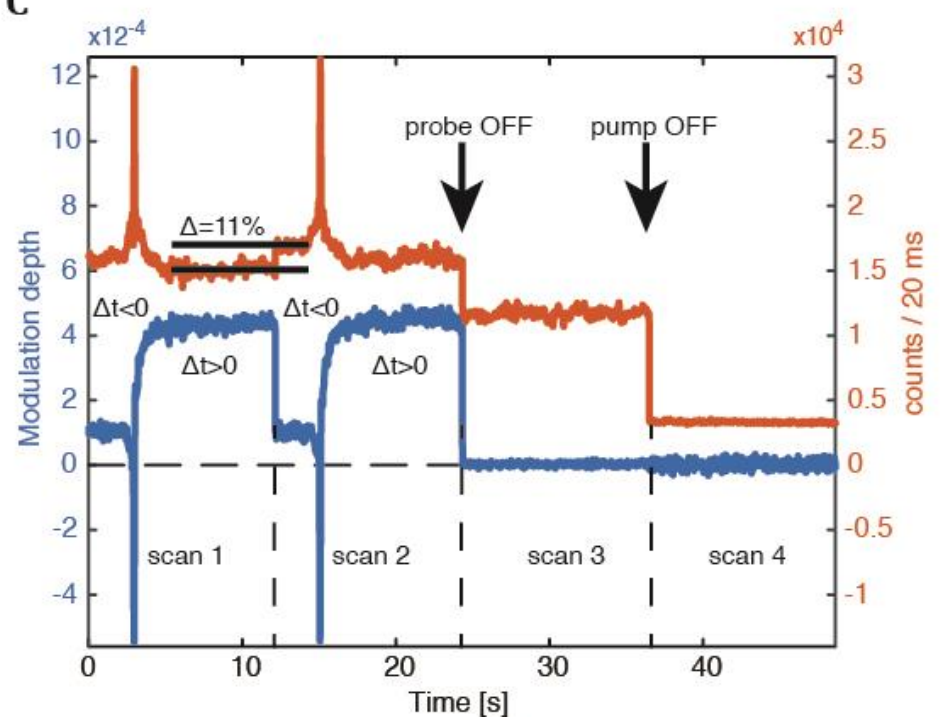

Supplementary Figure S7. Verifying the sign of the transient absorption signal.

(A) Intensity of the stimulation probe beam detected directly (no modulation applied) with a large area photodiode (PDA36A, Thorlabs). The two arrows indicate time points at which pump beam was switched $\mathrm{OFF}$ and $\mathrm{ON}$, respectively. A clear increase in the stimulation beam intensity is observed (red colored parts) when the pump beam is present. (B) Lock-in signal (averaged from a $5 \mathrm{~s}$ long time trace) as a function of pump modulation frequency ranging from $125 \mathrm{kHz}$ to $2 \mathrm{MHz}$. Error bars indicate standard deviations of the measurements. (C) Simultaneously recorded SE and PL signal as a function of time, while scanning the interpulse delay time $\Delta \mathrm{t}$ (4 consecutive scans). During scans 1-2, both beams are present. For this particular measurement we obtained PL depletion of $\sim 11 \%$ and stimulation beam modulation depth of $\sim 4 \cdot 10^{-4}$. At the beginning of scan 3 and 4 , the probe and pump beam was switched $\mathrm{OFF}$, respectively. SE signal vanishes whenever pump or probe beam is blocked. 
A

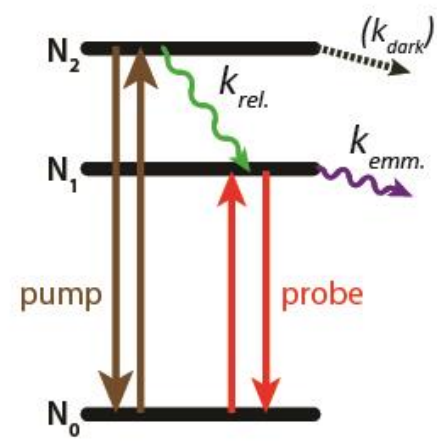

B

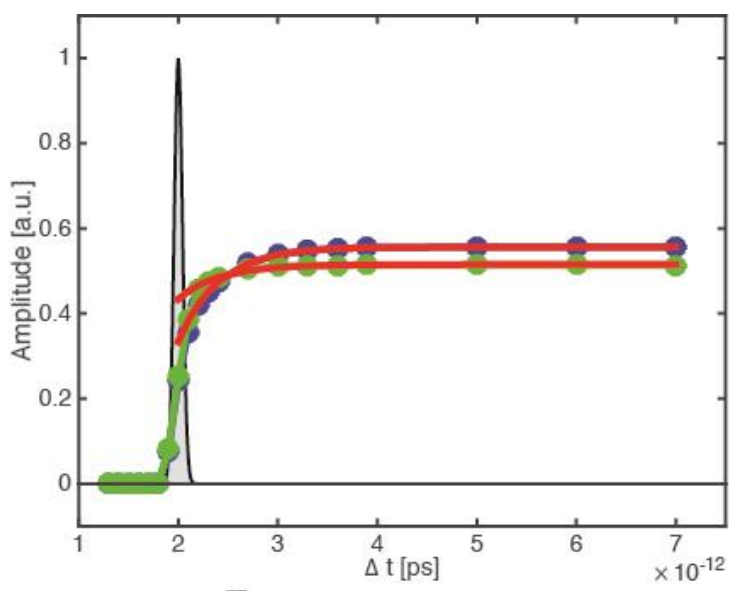

D

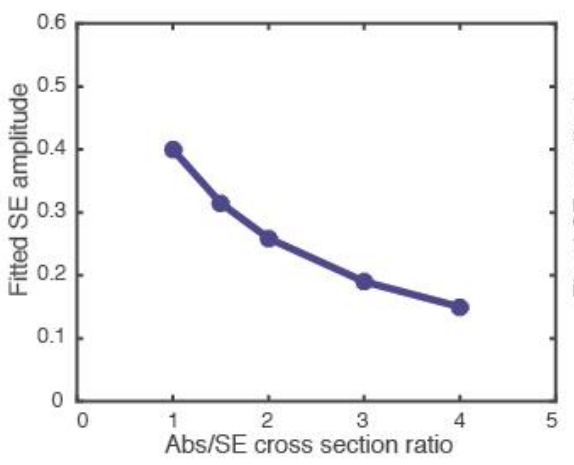

E

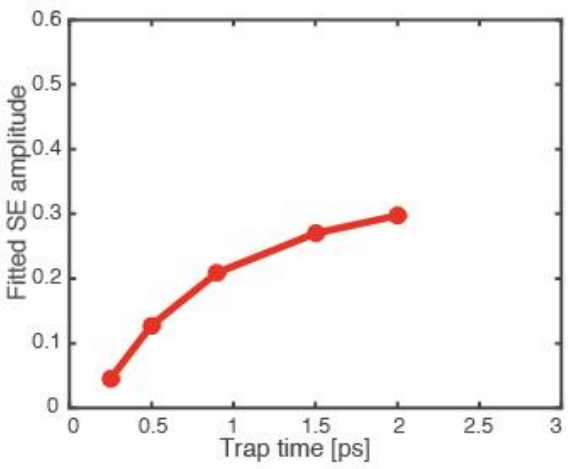

Supplementary Figure S8. Modelling the $\boldsymbol{S}_{\text {mod }}$ traces. (A) Three-state energy level diagram used as a model for simulating the $S_{\text {mod }}$ traces. (B) Grey profile represents the experimental excitation pulse. Green and blue traces are the results of solving the kinetic rate equations described in the Supplementary Text 6. Green trace includes relaxation of the excited charges to dark (trap) state and the absorption crosssection higher by $50 \%$ than SE cross-section. (C) Fitted SE/ $\mathrm{S}_{\bmod }$ as a function of fraction of the experimental pump and probe pulse intensity. Fraction equal to 1 corresponds to experimental conditions. (D) Fitted SE/ $\mathrm{S}_{\text {mod }}$ as a function of absorption to stimulated emission cross-section ratio. (E) Fitted SE $/ S_{\bmod }$ as a function of relaxation time to a dark state (trapping time $1 / k_{\text {dark }}$ ).

\section{References 26-36}

26. N. Accanto et al., Light Sci. Appl. 3, e143 (2014)

27. S. Berciaud et al., Phys. Rev. B 73, 045424 (2006)

28. M. Allione et al., ACS Nano 7, 2443-2452 (2013)

29. D. J. Norris, M. G. Bawendi, Phys. Rev. B 53, 16338-16346 (1996)

30. S. Christodoulou et al., J. Mater. Chem. C 2, 3439-3447 (2014)

31. M. G. Lupo et al., Nano Lett. 8, $4582-4587$ (2008)

32. G. Xing et al., ACS Nano 6, 10835-10844 (2012)

33. C. Galland et al., Nano Lett. 13, 321-328 (2013)

34. B. T. Diroll et al., Chem. Phys. Chem. 17, 759-765 (2016)

35. T. Walz et al., J. Mol. Biol. 282, 833-845 (1998)

36. L. Piatkowski, E. Gellings, N. F. van Hulst, Nat. Commun. 7, 10411 (2016) 